\title{
Parameters Estimation for the Exponentiated Weibull Distribution Based on Generalized Progressive Hybrid Censoring Schemes
}

\author{
Ahmed Elshahhat* \\ Department of Accounting \& Quantitative Information Systems, Faculty of Technology \& Development, Zagazig University, Egypt \\ *Corresponding author: dr_ahmed_elshahhat@yahoo.com
}

\begin{abstract}
Based on Type-I and Type-II generalized progressive hybrid censoring schemes, the maximum likelihood estimators and Bayes estimators for the unknown parameters of exponentiated Weibull lifetime model are derived. The approximate asymptotic variance-covariance matrix and approximate confidence intervals based on the asymptotic normality of the classical estimators are obtained. Independent non-informative types of priors are considered for the unknown parameters to develop the Bayes estimators and corresponding Bayes risks under a squared error loss function. Proposed estimators cannot be expressed in closed forms and can be evaluated numerically by some suitable iterative procedure. Finally, one real data set is analyzed for illustrative purposes.
\end{abstract}

Keywords: asymptotic variance-covariance matrix, Bayes estimator, confidence interval, exponentiated Weibull distribution, generalized progressive hybrid censoring schemes, maximum likelihood estimator, squared error loss function

Cite This Article: Ahmed Elshahhat, "Parameters Estimation for the Exponentiated Weibull Distribution Based on Generalized Progressive Hybrid Censoring Schemes." American Journal of Applied Mathematics and Statistics, vol. 5, no. 2 (2017): 33-48. doi: 10.12691/ajams-5-2-1.

\section{Introduction}

Many studies have considered censored samples which are Type-I, Type-II, hybrid and progressive hybrid censoring scheme. The exponentiated-Weibull distribution has been utilized for the analysis of life testing and reliability data. Recently, progressive hybrid censoring schemes (PHCSs) have become quite popular in a life testing problems and reliability analysis. Kundu and Joarder [6] proposed a PHCS, which is a mixture of Type-II progressive and hybrid censoring schemes, Childs et al. [2] refer to this censoring scheme as a Type-I PHCS and proposed Type-II PHCS to overcome the obvious drawback of the Type-I PHCS, that is maximum likelihood estimator (MLE) may not always exist. Type-I and TypeII generalized progressive hybrid censoring schemes (GPHCSs) proposed to overcome the drawbacks of the Type-I PHCS and Type-II PHCS, respectively. Cho et al. [3] proposed a new censoring scheme called GPHCS to overcome the drawback of the Type-I PHCS. One limitation of the Type-I PHCS, that is cannot be applied it when very few failures may occur before time $T$ accordingly the MLE for a parameter of underling lifetime model of observations may not be computed or its accuracy will be extremely low. Therefore, Cho et al. [3] suggested this Type of censoring to allow the experiment to continue beyond time $T$ and observed a pre-specified number of failures if very few failures had been observed up to time $T$. Under GPHCS the experimenter would ideally like to observe $m$-th failures, but is willing to accept a bare minimum of $k$-th failures. Lee et al. [8] refer to the GPHCS as a Type-I GPHCS and proposed the Type-II GPHCS to overcome the drawbacks in Type-II PHCS is that it might take a very long time to observe $m$-th failures and complete the life test. Type-II GPHCS is a modified for Type-II PHCS by guaranteeing that the test will be completed at time $T_{2}$, therefore, $T_{2}$ represents the absolute longest time that the researcher is willing to allow the experiment to continue. They suggested this type of censoring scheme to a guarantee the experiment terminated at a pre-fixed time.

Some recent studies on Type-I and Type-II GPHCSs have been carried out by many authors, including, Cho et al. [3] considered the Bayesian and maximum likelihood estimations for the entropy of Weibull distribution based on Type-I GPHCS. Cho et al. [4] obtained the exact distribution of the MLE as well as exact lower confidence bound for the exponential parameter under Type-I GPHCS. Lee et al. [7] obtained the MLE for the unknown parameter of exponential distribution under Type-II GPHCS. They derived to the exact and approximate conditional inference for the proposed estimator under Type-II GPHCS. Lee et al. [8] derived to exact inference of the unknown parameters under the assumptions that the lifetime distributions of different causes are independent identically distributed (IID) exponential competing risks model under Type-I GPHCS. Ashour and Elshahhat [1] obtained the MLEs and Bayes estimators for the unknown parameters of Weibull distribution based on Type-II 
GPHCS as well as they developed Bayes estimates and Bayes risks under a square error loss (SEL) function.

The rest of the paper is organized as follows: In Section 2, Type-I and Type-II GPHCSs are described. In Section 3, the MLEs are introduced for the unknown parameters of exponentiated Weibull (EW) distribution under Type-I and Type-II GPHCSs as well as some special cases are given, approximate asymptotic variance-covariance (V-Cov) matrix and approximate confidence interval (CI) for the MLEs using asymptotic distribution are obtained. In Section 4, the Bayes estimators and Bayes risks are developed under a SEL function and using independent non-informative priors for the unknown parameters of EW distribution based on Type-I and Type-II GPHCSs. It is clear that, the Bayesian and non-Bayesian estimators for EW parameters are not in closed forms, therefore, in Section 5, one numerical example is considered to illustrate the proposed estimators by using MathCad package version 14. Finally, we conclude the paper in Section 6.

\section{Model Description and Notation}

Type-I and Type-II GPHCSs are proposed to overcome the drawbacks of the Type-I and Type-II PHCSs, respectively, and they can be described as follows

\subsection{Type-I GPHCS}

This censoring scheme proposed by Cho et al. [3] and can be described as follows: Consider a life-testing experiment in which $n$ identical units are put to testing. Assume that $X_{(1)}, X_{(2)}, \ldots, X_{(n)}$ denote the corresponding lifetimes from a distribution with cumulative distribution function (CDF), $F(x)$, and probability density function (PDF), $f(x)$. The fix integers $k, m \in\{1,2, \ldots, n\}$ are prefixed, such that $k<m$ and $R_{j}, j=1,2, \ldots, m$, are pre-fixed integers which are satisfying $\sum_{j=1}^{m} R_{j}+m=n$ and
$T \in(0, \infty)$ is a pre-fixed time. At the time of first failure, $R_{1}$ of the remaining units are randomly removed. Similarly, at the time of the second failure, $R_{2}$ of the remaining units are removed, and so on. This process continues until to terminate at time $T^{*}=\max \left\{X_{(k)}, \min \left\{X_{(m)}, T\right\}\right\}$, at this time all of the remaining units are removed from the experiment. Let $D$ denote the number of observed failures up to time $T$. If the $m$-th failure occurs before time $T$, terminate the experiment at $X_{(m)}$ and the failures $X_{(k+1)}, \ldots, X_{(m)}$ are not observed. If the $m$-th failure occurs after $T$, or otherwise, the experiment terminate at $\max \left\{X_{(k)}, T\right\}$ as a schematic illustration in the Figure 1.

Based on the Type-I GPHCS, the observed data will be one of the following three forms:

$$
\begin{aligned}
& \text { Case-I : }\left\{X_{(1)}, X_{(2)}, \ldots, X_{(k)}\right\}, \quad \text { if } T<X_{(k)}<X_{(m)}, \\
& \text { Case-II }:\left\{X_{(1)}, X_{(2)}, \ldots, X_{(k)}, \ldots, X_{(D)}\right\}, \text { if } X_{(k)}<T<X_{(m)}, \\
& \text { Case-III }:\left\{X_{(1)}, X_{(2)}, \ldots, X_{(k)}, \ldots, X_{(m)}\right\}, \text { if } X_{(k)}<X_{(m)}<T .
\end{aligned}
$$

The likelihood function of the Type-I GPHCS can be written in the following form

$$
\begin{aligned}
& L_{i}(\theta \mid \underline{\mathrm{X}}) \\
& =\eta_{i} \prod_{j=1}^{V_{i}} f\left(x_{(j)} ; \theta\right)\left[1-F\left(x_{(j)} ; \theta\right)\right]^{R_{j}} A_{i}(T ; \theta),
\end{aligned}
$$

where, $i=1,2,3$, for Case-I,II and III, respectively, $V_{1}=k, V_{2}=D, V_{3}=m, A_{i}(T ; \theta)=1$, for $i=1,3$,

$$
\begin{aligned}
& A_{2}(T ; \theta)=[1-F(T ; \theta)]^{R_{D+1}^{*}}, R_{k}^{*}=n-k-\sum_{j=1}^{k-1} R_{j}, \\
& R_{D+1}^{*}=n-D-\sum_{j=1}^{D} R_{j} \text { and } \eta_{i}=\prod_{j=1}^{V_{i}} \sum_{k=j}^{m}\left(R_{k}+1\right) .
\end{aligned}
$$

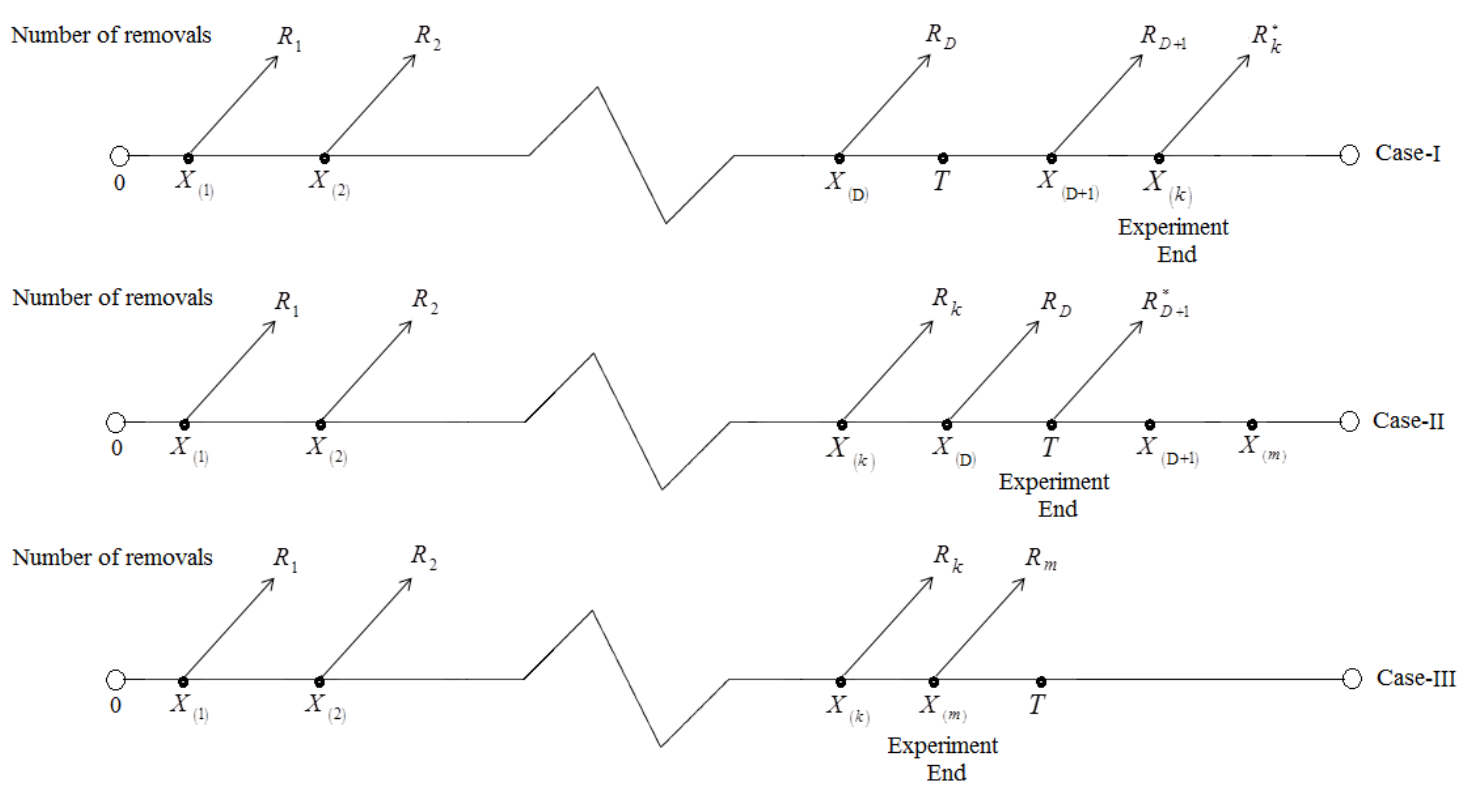

Figure 1. Schematic illustration of Type-I GPHCS 


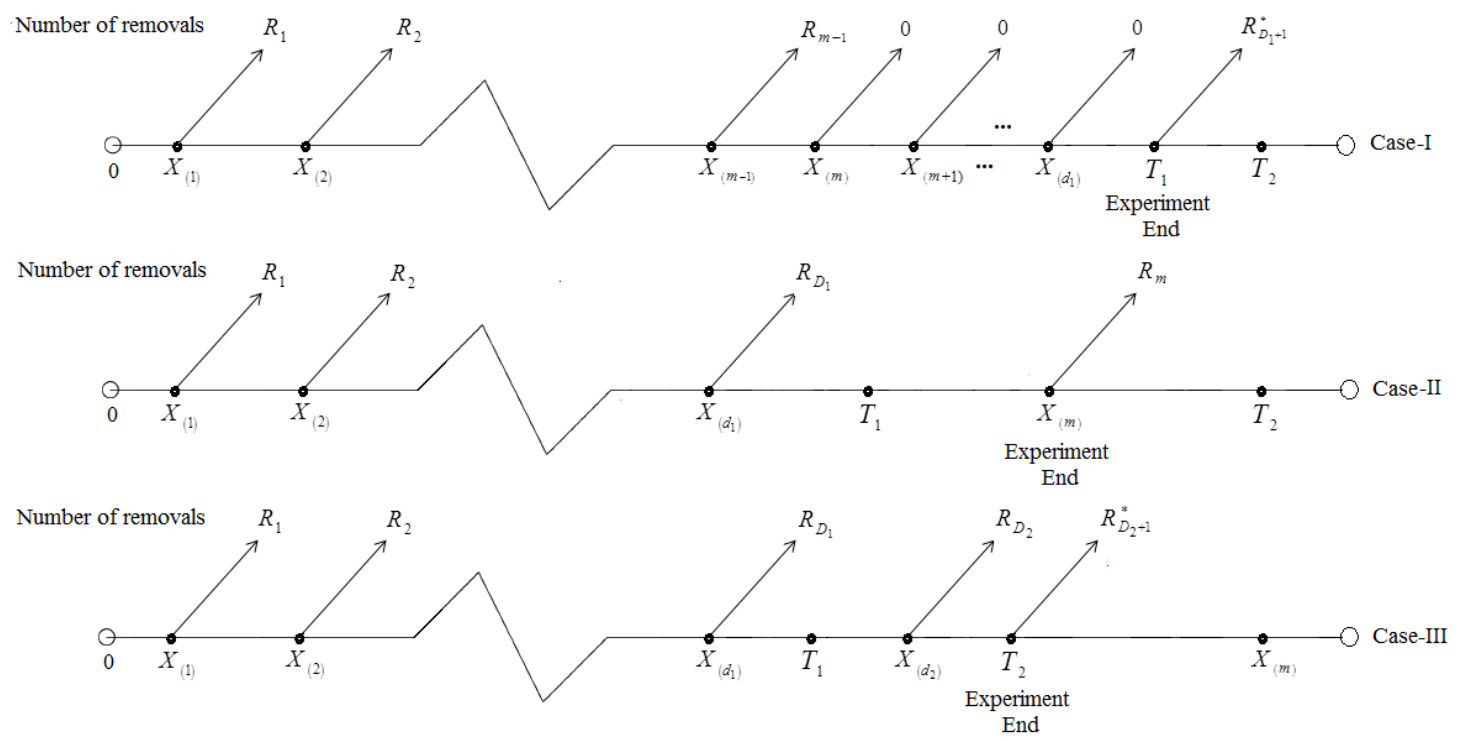

Figure 2. Schematic illustration of Type-II GPHCS

\subsection{Type-II GPHCS}

This censoring scheme proposed by Lee et al. [7] and can be described as follows: Consider a life test in which $n$ identical items are put on test. Assume that $X_{(1)}, X_{(2)}, \ldots, X_{(n)}$ denote the corresponding lifetimes from a distribution with $\operatorname{CDF} F(x)$ and $\operatorname{PDF} f(x)$. The integer $m$, times $T_{1}$ and $T_{2}$ are pre-assigned such that $m \leq n$ and $0<T_{1}<T_{2}<\infty$, and also $R_{1}, R_{2}, \ldots, R_{m}$ are pre-assigned integers satisfying $\sum_{j=1}^{m} R_{j}+m=n$. Let $D_{1}$ and $D_{2}$ denote the number of observed failures up to time $T_{1}$ and $T_{2}$, respectively. At the time of first observed failure, $R_{1}$ of the remaining items are withdrawn from the test at random. Following the second observed failure, $R_{2}$ of the remaining items are withdrawn and so on. This process continues until the termination time $T^{*}=\max \left\{T_{1}, \min \left\{X_{(m)}, T_{2}\right\}\right\}$, at this time all of the remaining units are removed from the experiment. If $X_{(m)}<T_{1}$, then instead of terminating the test by withdrawing the remaining $R_{m}$ items after the $m$-th failure, the experiment continue to observe failures but without any further withdrawals up to time $T_{1}$, therefore, $R_{j}=0$, for $j=m, m+1, \ldots, D_{1}$. If $\quad T_{1}<X_{(m)}<T_{2}$, terminate the test at $X_{(m)}$. If $X_{(m)}>T_{2}$, terminate the test at time $T_{2}$, as a schematic illustration is depicted in Figure 2.

Based on the Type-II GPHCS, the observed data will be one of the following three forms:

$$
\begin{array}{ll}
\text { Case-I }:\left\{X_{(1)}, \ldots, X_{(m)}, X_{(m+1)}, \ldots, X_{\left(d_{1}\right)}\right\}, \text { if } X_{(m)}<T_{1}<T_{2}, \\
\text { Case-II }:\left\{X_{(1)}, \ldots, X_{\left(d_{1}\right)}, \ldots, X_{(m)}\right\}, & \text { if } T_{1}<X_{(m)}<T_{2}, \\
\text { Case-III }:\left\{X_{(1)}, \ldots, X_{\left(d_{2}\right)}, \ldots, X_{(m)}\right\}, & \text { if } T_{1}<T_{2}<X_{(m)} .
\end{array}
$$

The likelihood function of the Type-II GPHCS can be written in the following form

$$
\begin{aligned}
& L_{i}(\theta \mid \underline{\mathrm{X}}) \\
& =\delta_{i} \prod_{j=1}^{S_{i}} f\left(x_{(j)} ; \theta\right)\left[1-F\left(x_{(j)} ; \theta\right)\right]^{R_{j}} A_{i}\left(T_{\tau} ; \theta\right),
\end{aligned}
$$

where, $i=1$, 2, 3, for Case-I, II and III, respectively, $S_{1}=D_{1}$, $S_{2}=m, \quad S_{3}=D_{2}, \quad A_{i}\left(T_{\tau} ; \theta\right)=\left[1-F\left(T_{\tau} ; \theta\right)\right]^{R_{D_{\tau}+1}^{*}}$ for $i=1,3$, and $\tau=1,2$, respectively, $A_{2}\left(T_{\tau} ; \theta\right)=1$, $R_{D_{1}+1}^{*}=n-d_{1}-\sum_{j=1}^{m-1} R_{j} \quad, \quad R_{D_{2}+1}^{*}=n-d_{2}-\sum_{j=1}^{d_{2}} R_{j}$ and $\delta_{i}=\prod_{j=1}^{S_{i}} \sum_{k=j}^{m}\left(R_{k}+1\right)$.

\section{Maximum Likelihood Estimators}

EW family introduced by Mudholkar and Srivastava [9], which is a simple generalization of well-known twoparameter Weibull distribution and is obtained by introducing one additional shape parameter. The EW distribution has been applied in areas of reliability analysis, quality control, duration and failure time modeling. Mudholkar et al. [10] presented and illustrated some applications of the EW distribution in reliability and survival studies. Mudholkar and Hutson [11] illustrate usefulness of the EW distribution in modeling extreme value data using the floods and Nassar and Eissa [12] derived to expressions for the mode of the EW distribution.

Suppose that the observed failures are IID from threeparameter $E W(\alpha, \beta, \theta)$ distribution with PDF

$$
f(x ; \alpha, \beta, \theta)=\frac{\alpha \theta}{\beta^{\alpha}} x^{\alpha-1} e^{-\left(\frac{x}{\beta}\right)^{\alpha}}\left(1-e^{-\left(\frac{x}{\beta}\right)^{\alpha}}\right)^{\theta-1},
$$

$$
x \geq 0, \alpha, \beta, \theta>0,
$$


and CDF is

$$
\begin{gathered}
F(x ; \alpha, \beta, \theta)=\left(1-e^{-\left(\frac{x}{\beta}\right)^{\alpha}}\right)^{\theta}, \\
x \geq 0, \alpha, \beta, \theta>0,
\end{gathered}
$$

where, $\alpha$ and $\theta$ both are the shape parameters and $\beta$ is the scale parameter.

The EW distribution has a two very well-known lifetime distributions as a special cases, if $\alpha=1$ and $\theta=1$, the EW distribution (3) reduced to exponential distribution with $\beta$ is scale parameter and if $\theta=1$, the EW distribution reduced to Weibull distribution with twoparameter $\alpha$ is shape parameter and $\beta$ is scale parameter.

Assuming that the failure times of the experimental units are follows the three-parameter EW distribution with PDF (3) and CDF (4), then the MLEs of the EW parameters can be obtained under Type-I and Type-II GPHCSs, respectively, as follows:

\subsection{MLEs of EW Parameters Based on Type- I GPHCS}

Based on the PDF and the CDF of EW distribution (3) and (4), respectively, then the likelihood function of the Type-I GPHCS (1) will be

$$
\begin{aligned}
& L_{i}(\underline{\omega} \mid \underline{\mathrm{X}}) \\
& =\eta_{i}\left(\frac{\alpha \theta}{\beta^{\alpha}}\right)^{V_{i}} \prod_{j=1}^{V_{i}} x_{(j)}^{\alpha-1} e^{-\left(\frac{x(j)}{\beta}\right)^{\alpha}} \\
& \times\left[1-e^{-\left(\frac{x(j)}{\beta}\right)^{\alpha}}\right)^{\theta-1} \\
& \left.\times\left[1-e^{-\left(\frac{x(j)}{\beta}\right)^{\alpha}}\right]^{\theta}\right]^{R_{j}} A_{i}(T ; \underline{\omega}),
\end{aligned}
$$

where, $i=1,2,3, \quad \underline{\omega}$ is parameter vector of the EW distribution, i.e., $\underline{\omega}=(\alpha, \beta, \theta)$,

$V_{1}=k, \quad A_{1}(T ; \underline{\omega})=1, \quad R_{k}^{*}=n-k-\sum_{j=1}^{k-1} R_{j}$, for Case-I,

$V_{2}=D, A_{2}(T ; \underline{\omega})=\left[1-\left(1-e^{-\left(\frac{T}{\beta}\right)^{\alpha}}\right)^{\theta}\right]^{R_{D+1}^{*}}$,

$R_{D+1}^{*}=n-D-\sum_{j=1}^{D} R_{j}$,

for Case-II,

and

$V_{3}=m, \quad A_{3}(T ; \underline{\omega})=1$,

for Case-III.

Additionally, the corresponding log-likelihood function of (5) can be written as follows

$$
\begin{aligned}
l_{i}(\underline{\omega} \mid \underline{\mathrm{X}}) & \propto\left\{V_{i} \log \left(\frac{\alpha \theta}{\beta^{\alpha}}\right)+(\alpha-1) \sum_{j=1}^{V_{i}} \log x_{(j)}\right. \\
& -\sum_{j=1}^{V_{i}} U^{\alpha}+(\theta-1) \sum_{j=1}^{V_{i}} \log \left(1-e^{-(U)^{\alpha}}\right) \\
& \left.+\sum_{j=1}^{V_{i}} R_{j} \log \left[1-\left(1-e^{-(U)^{\alpha}}\right)^{\theta}\right]+W_{i}(T ; \underline{\omega})\right\},
\end{aligned}
$$

where, $i=1,2,3, U=\frac{x_{(j)}}{\beta}, Q=\frac{T}{\beta}$,

$W_{1}(T ; \underline{\omega})=0$,

for Case-I,

$W_{2}(T ; \underline{\omega})=R_{D+1}^{*} \log \left[1-\left(1-e^{-(Q)^{\alpha}}\right)^{\theta}\right], \quad$ for Case-II, and

$W_{3}(T ; \underline{\omega})=0$,

for Case-III.

Differentiating (6) with respect to $\alpha, \beta$ and $\theta$, respectively, we get

$$
\begin{aligned}
& \frac{\partial l_{i}(\underline{\omega} \mid \underline{X})}{\partial \alpha} \\
& =\frac{V_{i}}{\alpha}(1-\alpha \log \beta)+\sum_{j=1}^{V_{i}} \log x_{(j)}-\sum_{j=1}^{V_{i}} U^{\alpha} \log U \\
& +(\theta-1) \sum_{j=1}^{V_{i}} e^{-(U)^{\alpha}} U^{\alpha} \log U\left(1-e^{-(U)^{\alpha}}\right)^{-1} \\
& -\theta \sum_{j=1}^{V_{i}} R_{j} e^{-(U)^{\alpha}} U^{\alpha} \log (U)\left(1-e^{-(U)^{\alpha}}\right)^{\theta-1} \\
& \times\left[1-\left(1-e^{-(U)^{\alpha}}\right)^{\theta}\right]^{-1} \frac{\partial W_{i}(T ; \underline{\omega})}{\partial \alpha} \\
& \frac{\partial l_{i}(\underline{\omega} \mid \underline{\mathrm{X}})}{\partial \beta}=-\frac{\alpha V_{i}}{\beta}+\frac{\alpha}{\beta} \sum_{j=1}^{V_{i}} U^{\alpha} \\
& -\frac{\alpha(\theta-1)}{\beta} \sum_{j=1}^{V_{i}} e^{-(U)^{\alpha}} U^{\alpha}\left(1-e^{-(U)^{\alpha}}\right)^{-1} \\
& +\frac{\alpha \theta}{\beta} \sum_{j=1}^{V_{i}} R_{j} e^{-(U)^{\alpha}} U^{\alpha}\left(1-e^{-(U)^{\alpha}}\right)^{\theta-1} \\
& \times\left[1-\left(1-e^{-(U)^{\alpha}}\right)^{\theta}\right]^{-1}+\frac{\partial W_{i}(T ; \underline{\omega})}{\partial \beta}
\end{aligned}
$$

and

$$
\begin{aligned}
\frac{\partial l_{i}(\underline{\omega} \mid \underline{\mathrm{X}})}{\partial \theta} & =\frac{V_{i}}{\theta}+\sum_{j=1}^{V_{i}} \log \left(1-e^{-(U)^{\alpha}}\right) \\
& -\sum_{j=1}^{V_{i}} R_{j}\left(1-e^{-(U)^{\alpha}}\right)^{\theta} \log \left(1-e^{-(U)^{\alpha}}\right)(7) \\
& \times\left[1-\left(1-e^{-(U)^{\alpha}}\right)^{\theta}\right]^{-1}+\frac{\partial W_{i}(T ; \underline{\omega})}{\partial \theta} .
\end{aligned}
$$


American Journal of Applied Mathematics and Statistics

37

where, $i=1,2,3, W_{i}(T ; \underline{\omega})=0$, for $i=1,3$,

$$
\begin{aligned}
& \frac{\partial W_{2}(T ; \underline{\omega})}{\partial \alpha} \\
& =-\theta R_{D+1}^{*} e^{-(Q)^{\alpha}} Q^{\alpha} \\
& \quad \times \log Q\left(1-e^{\left.-(Q)^{\alpha}\right)^{\theta-1}\left[1-\left(1-e^{-(Q)^{\alpha}}\right)^{\theta}\right]^{-1},}\right. \\
& \frac{\partial W_{2}(T ; \underline{\omega})}{\partial \beta}=\frac{\alpha \theta}{\beta} R_{D+1}^{*} e^{-(Q)^{\alpha}} Q^{\alpha}\left(1-e^{-(Q)^{\alpha}}\right)^{\theta-1} \\
& \times\left[1-\left(1-e^{-(Q)^{\alpha}}\right)^{\theta}\right]^{-1},
\end{aligned}
$$

and

$$
\begin{aligned}
\frac{\partial W_{2}(T ; \underline{\omega})}{\partial \theta}= & -R_{D+1}^{*}\left(1-e^{-(Q)^{\alpha}}\right)^{\theta} \\
& \times \log \left(1-e^{-(Q)^{\alpha}}\right)\left[1-\left(1-e^{-(Q)^{\alpha}}\right)^{\theta}\right]^{-1} .
\end{aligned}
$$

Equating the first derivations (7) to zero and solving for $\hat{\alpha}, \hat{\beta}$ and $\hat{\theta}$, we get the ALEs $\hat{\alpha}, \hat{\beta}$ and $\hat{\theta}$ of $\alpha, \beta$ and $\theta$ based on Type-I GPHCS, respectively, in the following forms

$$
\begin{aligned}
& \hat{\alpha}_{i}\left(\hat{\beta}_{i}, \hat{\theta}_{i}\right) \\
& =V_{i}\left(1-\hat{\alpha}_{i} \log \hat{\beta}_{i}\right)\left\{\sum_{j=1}^{V_{i}} U^{\hat{\alpha}_{i}} \log U-\sum_{j=1}^{V_{i}} \log x_{(j)}\right. \\
& -\left(\hat{\theta}_{i}-1\right) \sum_{j=1}^{V_{i}} e^{-(U)^{\hat{\alpha}_{i}}} U^{\hat{\alpha}_{i}} \log U \times\left(1-e^{-(U)^{\hat{\alpha}_{i}}}\right)^{-1} \\
& +\hat{\theta}_{i} \sum_{j=1}^{V_{i}} R_{j} e^{-(U)^{\hat{\alpha}_{i}}} U^{\hat{\alpha}_{i}} \log (U)\left(1-e^{-(U)^{\hat{\alpha}_{i}}}\right)^{\hat{\theta}_{i}-1} \\
& \left.\times\left[1-\left(1-e^{-(U)^{\hat{\alpha}_{i}}}\right)^{\hat{\theta}_{i}}\right]^{-1}-\frac{\partial W_{i}(T ; \hat{\hat{\omega}})}{\partial \hat{\alpha}_{i}}\right\}^{-1} . \\
& \hat{\beta}_{i}\left(\hat{\alpha}_{i}, \hat{\theta}_{i}\right) \\
& =\hat{\alpha}_{i} V_{i}\left\{\frac{\hat{\alpha}_{i}}{\hat{\beta}_{i}} \sum_{j=1}^{V_{i}} U^{\hat{\alpha}_{i}}\right. \\
& -\frac{\hat{\alpha}_{i}\left(\hat{\theta}_{i}-1\right)}{\hat{\beta}_{i}} \sum_{j=1}^{V_{i}} e^{-(U)^{\hat{\alpha}_{i}}} U^{\hat{\alpha}_{i}}\left(1-e^{-(U)^{\hat{\alpha}_{i}}}\right)^{-1} \\
& +\frac{\hat{\alpha}_{i} \hat{\theta}_{i}}{\hat{\beta}_{i}} \sum_{j=1}^{V_{i}} R_{j} e^{-(U)^{\hat{\alpha}_{i}}} U^{\hat{\alpha}_{i}}\left(1-e^{-(U)^{\hat{\alpha}_{i}}}\right)^{\hat{\theta}_{i}-1} \\
& \left.\times\left[1-\left(1-e^{-(U)^{\hat{\alpha}_{i}}}\right)^{\hat{\theta}_{i}}\right]^{-1}+\frac{\partial W_{i}(T ; \underline{\hat{\omega}})}{\partial \hat{\beta}_{i}}\right\}^{-1} .
\end{aligned}
$$

and

$$
\begin{aligned}
& \hat{\theta}_{i}\left(\hat{\alpha}_{i}, \hat{\beta}_{i}\right) \\
& =V_{i}\left\{\sum_{j=1}^{V_{i}} R_{j}\left(1-e^{-(U)^{\hat{\alpha}_{i}}}\right)^{\hat{\theta}_{i}}\right. \\
& \times \log \left(1-e^{-(U)^{\hat{\alpha}_{i}}}\right)\left[1-\left(1-e^{-(U)^{\hat{\alpha}_{i}}}\right)^{\hat{\theta}_{i}}\right]^{-1} \\
& \left.-\sum_{j=1}^{V_{i}} \log \left(1-e^{-(U)^{\hat{\alpha}_{i}}}\right)-\frac{\partial W_{i}(T ; \underline{\hat{\omega}})}{\partial \hat{\theta}_{i}}\right\}^{-1} .
\end{aligned}
$$

where, $i=1,2,3, \quad U=\frac{x_{(j)}}{\hat{\beta}_{i}}, Q=\frac{T}{\hat{\beta}_{i}}, W_{i}(T ; \underline{\hat{\omega}})=0$, for $i=1,3$,

$$
\begin{aligned}
& \frac{\partial W_{2}(T ; \underline{\hat{\omega}})}{\partial \hat{\alpha}_{i}} \\
& =-\hat{\theta}_{i} R_{D+1}^{*} e^{-(Q)^{\hat{\alpha}_{i}}} Q^{\hat{\alpha}_{i}} \\
& \times \log Q\left(1-e^{-(Q)^{\hat{\alpha}_{i}}}\right)^{\hat{\theta}_{i}-1}\left[1-\left(1-e^{-(Q)^{\hat{\alpha}_{i}}}\right)^{\hat{\theta}_{i}}\right]^{-1}, \\
& \quad \frac{\partial W_{2}(T ; \underline{\hat{\omega}})}{\partial \hat{\beta}_{i}}, \\
& =\frac{\hat{\alpha}_{i} \hat{\theta}_{i}}{\hat{\beta}_{i}} R_{D+1}^{*} e^{-(Q)^{\hat{\alpha}_{i}}} Q^{\hat{\alpha}_{i}}\left(1-e^{-(Q)^{\hat{\alpha}_{i}}}\right)^{\hat{\theta}_{i}-1} \\
& \quad\left[1-\left(1-e^{-(Q)^{\hat{\alpha}_{i}}}\right)^{\hat{\theta}_{i}}\right]^{-1},
\end{aligned}
$$

and

$$
\begin{aligned}
& \frac{\partial W_{2}(T ; \underline{\hat{\omega}})}{\partial \hat{\theta}_{i}} \\
& =-R_{D+1}^{*}\left(1-e^{-(Q)^{\hat{\alpha}_{i}}}\right)^{\hat{\theta}_{i}} \log \left(1-e^{-(Q)^{\hat{\alpha}_{i}}}\right) \\
& \times\left[1-\left(1-e^{-(Q)^{\hat{\alpha}_{i}}}\right)^{\hat{\theta}_{i}}\right]^{-1} .
\end{aligned}
$$

Clearly, the ALEs $\hat{\alpha}, \hat{\beta}$ and $\hat{\theta}$ of EW parameters $\alpha, \beta$ and $\theta$ based on Type-I GPHCS, respectively, can be obtained by solving set of nonlinear equations, this needs computer facilities and numerical techniques. Also, Chr et al. [4] results can be obtained as a special case from (6), if putting $\alpha=\theta=1$, we get the MLE $\hat{\beta}$ in the case of exponential parameter $\beta$, if putting $\theta=1$, we get the ALEs $\hat{\alpha}$ and $\hat{\beta}$ in the case of Weibull parameters $\alpha$ and $\beta$, respectively. 


\subsection{MLEs of EW Parameters Based on Type-II GPHCS}

Based on the PDF and the CDF of EW distribution (3) and (4), respectively, then the likelihood function of the Type-II GPHCS (2) can be rewritten as follows:

$$
\begin{aligned}
& L_{i}(\underline{\omega} \mid \underline{\mathrm{X}}) \\
& =\delta_{i}\left(\frac{\alpha \theta}{\beta^{\alpha}}\right)^{S_{i}} \prod_{j=1}^{S_{i} x_{(j)}^{\alpha-1} e^{-\left(\frac{x(j)}{\beta}\right)^{\alpha}}} 1^{\left.-\left(\frac{x(j)}{\beta}\right)^{\alpha}\right)^{\theta-1}} \\
& \times\left[1-\left(1-e^{-\left(\frac{x(j)}{\beta}\right)^{\alpha}}\right)^{\theta}\right]^{R_{j}} A_{i}\left(T_{\tau} ; \underline{\omega}\right),
\end{aligned}
$$

where, $i=1,2,3, \quad \underline{\omega}$ is parameter vector of $\mathrm{EW}$ distribution, i.e., $\underline{\omega}=(\alpha, \beta, \theta)$,

$S_{1}=D_{1}, \quad A_{1}\left(T_{1} ; \underline{\omega}\right)=\left[1-\left(1-e^{-\left(\frac{T_{1}}{\beta}\right)^{\alpha}}\right)^{\theta}\right]^{R_{D_{1}+1}^{*}}$,

$R_{D_{1}+1}^{*}=n-d_{1}-\sum_{i=1}^{m-1} R_{i}$,

for Case-I,

$S_{2}=m, \quad A_{2}\left(T_{\tau} ; \underline{\omega}\right)=1$,

for Case-II,

and

$S_{3}=D_{2}, \quad A_{3}\left(T_{2} ; \underline{\omega}\right)=\left[1-\left(1-e^{-\left(\frac{T_{2}}{\beta}\right)^{\alpha}}\right)^{\theta}\right]^{R_{D_{2}+1}^{*}}$,

$R_{D_{2}+1}^{*}=n-d_{2}-\sum_{i=1}^{d_{2}} R_{i}$,

for Case-III.

Additionally, the corresponding log-likelihood function of (8) will be

$$
\begin{aligned}
l_{i}(\underline{\omega} \mid \underline{\mathrm{X}}) \propto & \left\{S_{i} \log \left(\frac{\alpha \theta}{\beta^{\alpha}}\right)+(\alpha-1) \sum_{j=1}^{S_{i}} \log x_{(j)}\right. \\
& -\sum_{j=1}^{S_{i}} U^{\alpha}+(\theta-1) \sum_{j=1}^{S_{i}} \log \left(1-e^{-(U)^{\alpha}}\right)(9) \\
& +\sum_{j=1}^{S_{i}} R_{j} \log \left[1-\left(1-e^{-(U)^{\alpha}}\right)^{\theta}\right] \\
& \left.+W_{i}\left(T_{\tau} ; \underline{\omega}\right)\right\},
\end{aligned}
$$

where, $\quad i=1,2,3, \quad U=\frac{x_{(j)}}{\beta}, \quad \tau=1,2, \quad$ for $\quad i=1,3$, respectively,
$W_{1}\left(T_{1} ; \underline{\omega}\right)=R_{D_{1}+1}^{*} \log \left[1-\left(1-e^{-\left(\frac{T_{1}}{\beta}\right)^{\alpha}}\right)^{\theta}\right]$, for Case-I,

$W_{2}\left(T_{\tau} ; \underline{\omega}\right)=0$,

for Case-II,

and

$W_{3}\left(T_{2} ; \underline{\omega}\right)=R_{D_{2}+1}^{*} \log \left[1-\left(1-e^{-\left(\frac{T_{2}}{\beta}\right)^{\alpha}}\right)^{\theta}\right]$, for Case-III.

Differentiating (9) with respect to $\alpha, \beta$ and $\theta$, respectively, we get

$$
\begin{aligned}
& \frac{\partial l_{i}(\underline{\omega} \mid \underline{X})}{\partial \alpha} \\
= & \frac{S_{i}}{\alpha}(1-\alpha \log \beta)+\sum_{j=1}^{S_{i}} \log X_{(j)}-\sum_{j=1}^{S_{i}} U^{\alpha} \log U \\
+ & (\theta-1) \sum_{j=1}^{S_{i}} e^{-(U)^{\alpha}} U^{\alpha} \log U\left(1-e^{-(U)^{\alpha}}\right)^{-1} \\
& -\theta \sum_{j=1}^{S_{i}} R_{j} e^{-(U)^{\alpha}} U^{\alpha} \log U\left(1-e^{-(U)^{\alpha}}\right)^{\theta-1} \\
& \times\left[1-\left(1-e^{-(U)^{\alpha}}\right)^{\theta}\right]^{-1}+\frac{\partial W_{i}\left(T_{\tau} ; \underline{\omega}\right)}{\partial \alpha} .
\end{aligned}
$$

$$
\begin{aligned}
& \frac{\partial l_{i}(\underline{\omega} \mid \underline{\mathrm{X}})}{\partial \beta} \\
& =-\frac{\alpha S_{i}}{\beta}+\frac{\alpha}{\beta} \sum_{j=1}^{S_{i}} U^{\alpha} \\
& -\frac{\alpha(\theta-1)}{\beta} \sum_{j=1}^{S_{i}} e^{-(U)^{\alpha}} U^{\alpha}\left(1-e^{-(U)^{\alpha}}\right)^{-1} \\
& +\frac{\alpha \theta}{\beta} \sum_{j=1}^{S_{i}} R_{j} e^{-(U)^{\alpha}} U^{\alpha}\left(1-e^{-(U)^{\alpha}}\right)^{\theta-1} \\
& \quad \times\left[1-\left(1-e^{\left.\left.-(U)^{\alpha}\right)^{\theta}\right]^{-1}+\frac{\partial W_{i}\left(T_{\tau} ; \underline{\omega}\right)}{\partial \beta} .}\right.\right.
\end{aligned}
$$

and

$$
\begin{aligned}
& \frac{\partial l_{i}(\underline{\omega} \mid \underline{X})}{\partial \theta} \\
& =\frac{S_{i}}{\theta}+\sum_{j=1}^{S_{i}} \log \left(1-e^{-(U)^{\alpha}}\right) \\
& -\sum_{j=1}^{S_{i}} R_{j}\left(1-e^{-(U)^{\alpha}}\right)^{\theta} \log \left(1-e^{-(U)^{\alpha}}\right) \\
& \quad \times\left[1-\left(1-e^{-(U)^{\alpha}}\right)^{\theta}\right]^{-1}+\frac{\partial W_{i}\left(T_{\tau} ; \underline{\omega}\right)}{\partial \theta} .
\end{aligned}
$$


where, $i=1,2,3, \quad W_{2}\left(T_{\tau} ; \underline{\omega}\right)=0, \quad Q=\frac{T_{\tau}}{\beta}, \tau=1,2$, for $i=1,3$, respectively,

$$
\begin{aligned}
& \frac{\partial W_{i}\left(T_{\tau} ; \underline{\omega}\right)}{\partial \alpha}=-\theta R_{D_{\tau}+1}^{*} e^{-(Q)^{\alpha}} Q^{\alpha} \log Q \\
& \times\left(1-e^{-(Q)^{\alpha}}\right)^{\theta-1}\left[1-\left(1-e^{-(Q)^{\alpha}}\right)^{\theta}\right]^{-1}, \\
& \frac{\partial W_{i}\left(T_{\tau} ; \underline{\omega}\right)}{\partial \beta}=\frac{\alpha \theta}{\beta} R_{D_{\tau}+1}^{*} e^{-(Q)^{\alpha}} Q^{\alpha} \\
& \times\left(1-e^{-(Q)^{\alpha}}\right)^{\theta-1}\left[1-\left(1-e^{-(Q)^{\alpha}}\right)^{\theta}\right]^{-1},
\end{aligned}
$$

and

$$
\begin{aligned}
\frac{\partial W_{i}\left(T_{\tau} ; \underline{\omega}\right)}{\partial \theta}= & -R_{D_{\tau}+1}^{*}\left(1-e^{-(Q)^{\alpha}}\right)^{\theta} \\
& \times \log \left(1-e^{-(Q)^{\alpha}}\right)\left[1-\left(1-e^{-(Q)^{\alpha}}\right)^{\theta}\right]^{-1} .
\end{aligned}
$$

Equating the first derivations (10) to zero and solving for $\hat{\alpha}, \hat{\beta}$ and $\hat{\theta}$, we get the MLEs $\hat{\alpha}, \hat{\beta}$ and $\hat{\theta}$ of $\alpha, \beta$ and $\theta$ based on Type-II GPHCS, respectively, as in the following forms

$$
\begin{aligned}
& \hat{\alpha}_{i}\left(\hat{\beta}_{i}, \hat{\theta}_{i}\right) \\
& =S_{i}\left(1-\hat{\alpha}_{i} \log \hat{\beta}_{i}\right)\left\{\sum_{j=1}^{S_{i}} U^{\hat{\alpha}_{i}} \log U-\sum_{j=1}^{S_{i}} \log x_{(j)}\right. \\
& -\left(\hat{\theta}_{i}-1\right) \sum_{j=1}^{S_{i}} e^{-(U)^{\hat{\alpha}_{i}}} U^{\hat{\alpha}_{i}} \log U\left(1-e^{-(U)^{\hat{\alpha}_{i}}}\right)^{-1} \\
& +\hat{\theta}_{i} \sum_{j=1}^{S_{i}} R_{j} e^{-(U)^{\hat{\alpha}_{i}}} U^{\hat{\alpha}_{i}} \log (U)\left(1-e^{-(U)^{\hat{\alpha}_{i}}}\right)^{\hat{\theta}_{i}-1} \\
& \left.\times\left[1-\left(1-e^{-(U)^{\hat{\alpha}_{i}}}\right)^{\hat{\theta}_{i}}\right]^{-1}-\frac{\partial W_{i}\left(T_{\tau} ; \hat{\hat{\omega}}\right)}{\partial \hat{\alpha}_{i}}\right\}^{-1} . \\
& \hat{\beta}_{i}\left(\hat{\alpha}_{i}, \hat{\theta}_{i}\right) \\
& =\hat{\alpha}_{i} S_{i}\left\{\frac{\hat{\alpha}_{i}}{\hat{\beta}_{i}} \sum_{j=1}^{S_{i}} U^{\hat{\alpha}_{i}}\right. \\
& -\frac{\hat{\alpha}_{i}\left(\hat{\theta}_{i}-1\right)}{\hat{\beta}_{i}} \sum_{j=1}^{S_{i}} e^{-(U)^{\hat{\alpha}_{i}}} U^{\hat{\alpha}_{i}}\left(1-e^{-(U)^{\hat{\alpha}_{i}}}\right)^{-1} \\
& +\frac{\hat{\alpha}_{i} \hat{\theta}_{i}}{\hat{\beta}_{i}} \sum_{j=1}^{S_{i}} R_{j} e^{-(U)^{\hat{\alpha}_{i}}} U^{\hat{\alpha}_{i}}\left(1-e^{-(U)^{\hat{\alpha}_{i}}}\right)^{\hat{\theta}_{i}-1} \\
& \left.\times\left[1-\left(1-e^{-(U)^{\hat{\alpha}_{i}}}\right)^{\hat{\theta}_{i}}\right]^{-1}+\frac{\partial W_{i}\left(T_{\tau} ; \hat{\hat{\omega}}\right)}{\partial \hat{\beta}_{i}}\right\}^{-1} .
\end{aligned}
$$

and

$$
\begin{aligned}
& \hat{\theta}_{i}\left(\hat{\alpha}_{i}, \hat{\beta}_{i}\right) \\
& =S_{i}\left\{\sum_{j=1}^{S_{i}} R_{j}\left(1-e^{-(U)^{\hat{\alpha}_{i}}}\right)^{\hat{\theta}_{i}}\right. \\
& \times \log \left(1-e^{-(U)^{\hat{\alpha}_{i}}}\right)\left[1-\left(1-e^{-(U)^{\hat{\alpha}_{i}}}\right)^{\hat{\theta}_{i}}\right]^{-1} \\
& \left.-\sum_{j=1}^{S_{i}} \log \left(1-e^{-(U)^{\hat{\alpha}_{i}}}\right)-\frac{\partial W_{i}\left(T_{\tau} ; \underline{\hat{\omega}}\right)}{\partial \hat{\theta}_{i}}\right\}^{-1} .
\end{aligned}
$$

where, $i=1,2,3, \quad W_{2}\left(T_{\tau} ; \underline{\hat{\omega}}\right)=0, \quad U=\frac{x_{(j)}}{\hat{\beta}_{i}}, Q=\frac{T_{\tau}}{\hat{\beta}_{i}}$, $\tau=1,2$, for $i=1,3$, respectively,

$$
\begin{aligned}
\frac{\partial W_{i}\left(T_{\tau} ; \underline{\hat{\omega}}\right)}{\partial \hat{\alpha}_{i}} & =-\hat{\theta}_{i} R_{D_{\tau}+1}^{*} e^{-(Q)^{\hat{\alpha}_{i}}} Q^{\hat{\alpha}_{i}} \log Q \\
& \times\left(1-e^{-(Q)^{\hat{\alpha}_{i}}}\right)^{\hat{\theta}_{i}-1}\left[1-\left(1-e^{-(Q)^{\hat{\alpha}_{i}}}\right)^{\hat{\theta}_{i}}\right]^{-1},
\end{aligned}
$$

$$
\begin{aligned}
\frac{\partial W_{i}\left(T_{\tau} ; \underline{\hat{\omega}}\right)}{\partial \hat{\beta}_{i}} & =\frac{\hat{\alpha}_{i} \hat{\theta}_{i}}{\hat{\beta}_{i}} R_{D_{\tau}+1}^{*} e^{-(Q)^{\hat{\alpha}_{i}}} Q^{\hat{\alpha}_{i}} \\
& \times\left(1-e^{-(Q)^{\hat{\alpha}_{i}}}\right)^{\hat{\theta}_{i}-1}\left[1-\left(1-e^{-(Q)^{\hat{\alpha}_{i}}}\right)^{\hat{\theta}_{i}}\right]^{-1},
\end{aligned}
$$

and

$$
\begin{aligned}
\frac{\partial W_{i}\left(T_{\tau} ; \underline{\hat{\omega}}\right)}{\partial \hat{\theta}_{i}}= & -R_{D_{\tau}+1}^{*}\left(1-e^{-(Q)^{\hat{\alpha}_{i}}}\right)^{\hat{\theta}_{i}} \\
& \times \log \left(1-e^{-(Q)^{\hat{\alpha}_{i}}}\right)\left[1-\left(1-e^{-(Q)^{\hat{\alpha}_{i}}}\right)^{\hat{\theta}_{i}}\right]^{-1} .
\end{aligned}
$$

Clearly, the MLEs $\hat{\alpha}, \hat{\beta}$ and $\hat{\theta}$ of EW parameters $\alpha, \beta$ and $\theta$ based on Type-II GPHCS, respectively, do not result in closed forms, this needs computer facilities and numerical techniques to evaluated numerically. Some special cases can be obtained from (9), Lee et al. [7] results in the case of exponential distribution by putting $\alpha=\theta=1$. Also, Ashour and Elshahhat [1]) results in the case of Weibull distribution by putting $\theta=1$.

Asymptotic V-Cov matrix of the MLEs for EW parameters $\underline{\omega}$ can be obtained by inverting the Fisher information matrix $\mathrm{I}(\underline{\omega})$, which is can be obtained by taking the negative expectation for the second partial derivatives of the natural logarithm likelihood function as

$$
\mathrm{I}_{a, b}(\underline{\omega})=-E\left[\frac{\partial^{2} l_{i}(\underline{\omega} \mid \underline{\mathrm{X}})}{\partial^{2} \underline{\omega}}\right], \quad a, b=1,2,3 .
$$


Cohen [5] concluded that the approximate V-Cov matrix may be obtained by replacing expected values by their MLEs, i.e., estimating $\mathrm{I}_{0}^{-1}(\underline{\omega})$ by $\mathrm{I}_{0}^{-1}(\underline{\hat{\omega}})$, then

$$
\begin{aligned}
& \mathrm{I}_{0}^{-1}(\underline{\hat{\omega}})
\end{aligned}
$$

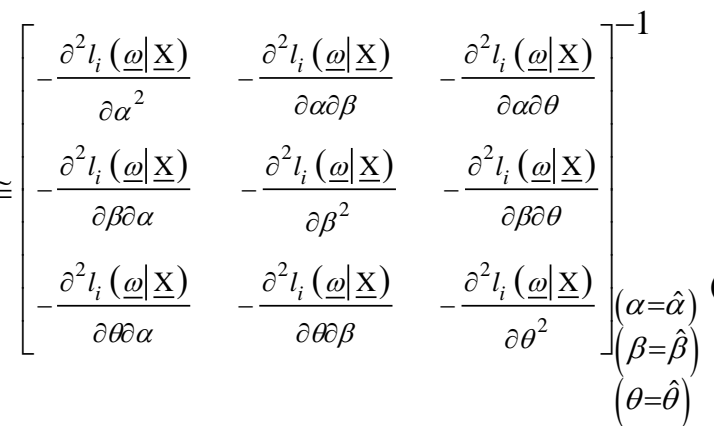

$$
\begin{aligned}
& =\left[\begin{array}{ccc}
\hat{\sigma}_{\alpha}^{2} & \hat{\sigma}_{\alpha, \beta} & \hat{\sigma}_{\alpha, \theta} \\
\hat{\sigma}_{\beta, \alpha} & \hat{\sigma}_{\beta}^{2} & \hat{\sigma}_{\beta, \theta} \\
\hat{\sigma}_{\theta, \alpha} & \hat{\sigma}_{\theta, \alpha} & \hat{\sigma}_{\theta}^{2}
\end{array}\right] .
\end{aligned}
$$

Based on the log-likelihood functions (6) and (9), the approximate asymptotic V-Cov matrix for the MLEs of the three-parameter EW distribution can be obtained based on Type-I and Type-II GPHCSs, respectively. The elements of the observed information matrix (11) are obtained based on the log-likelihood functions of Type-I GPHCS (6) and Type-II GPHCS (9) and reported in Appendix A and B, respectively.

Under the regularity conditions for the asymptotic properties of MLEs of EW parameters $\alpha, \beta$ and $\theta$, the asymptotic normality of the MLEs $\hat{\alpha}_{i}, \hat{\beta}_{i}$ and $\hat{\theta}_{i}$ is approximately multivariately normal, i.e., $\underline{\hat{\omega}} \sim N\left(\underline{\omega}, \mathrm{I}_{0}^{-1}(\underline{\hat{\omega}})\right)$. The $100(1-\varepsilon) \%$ approximate CIs for EW parameters $\alpha, \beta$ and $\theta$ based on Type-I and Type-II GPHCSs can be obtained using the asymptotic normality of the MLEs $\hat{\alpha}_{i}, \hat{\beta}_{i}$ and $\hat{\theta}_{i}$, respectively, as follows:

$$
\begin{aligned}
& \hat{\alpha}_{i} \pm z_{\varepsilon / 2} \cdot \sqrt{\hat{\sigma}_{\alpha}^{2}}, \hat{\beta}_{i} \pm z_{\varepsilon / 2} \cdot \sqrt{\hat{\sigma}_{\beta}^{2}} \\
& \text { and } \hat{\theta}_{i} \pm z_{\varepsilon / 2} \cdot \sqrt{\hat{\sigma}_{\theta}^{2}}, i=1,2,3
\end{aligned}
$$

where, $\hat{\sigma}_{\alpha}^{2}, \hat{\sigma}_{\beta}^{2}$ and $\hat{\sigma}_{\theta}^{2}$ are the elements on the main diagonal of the approximate asymptotic V-Cov matrix (11), respectively, and $z_{\varepsilon / 2}$ is the percentile of the standard normal distribution with upper probability $\varepsilon / 2-t h$.

\section{Bayes Estimators}

Following Singh et al. (2005), the Bayes estimators can be develop for the EW parameters $\alpha, \beta$ and $\theta$ based on Type-I and Type-II GPHCSs, we consider independent non-informative priors $g_{1}(\alpha), g_{2}(\beta)$ and $g_{3}(\theta)$, given as

$$
\begin{aligned}
& g_{1}(\alpha)=\frac{1}{c}, \quad c>\alpha>0, \\
& g_{2}(\beta)=\frac{1}{\beta}, \quad \beta>0,
\end{aligned}
$$

and

$$
g_{3}(\theta)=\frac{1}{\theta}, \quad \theta>0 .
$$

Using a very well-known symmetric loss function is the SEL function, $\ell(\theta, \tilde{\theta})=(\tilde{\theta}-\theta)^{2}$, to obtain the Bayes estimators for the three parameter of EW distribution under Type-I and Type-II GPHCSs. Under this loss function, the Bayes estimator is the posterior mean as well as the Bayes risk is the posterior variance. The Bayes estimator is defined as

$$
\tilde{\theta}=\int_{\Theta} \theta \cdot \pi(\theta \mid \underline{\mathrm{X}}) d \theta
$$

\subsection{Bayes Estimators of EW Parameters Based on Type-I GPHCS}

Based on the likelihood function (5), the noninformative priors (12) and using the Bayes theorem, the joint posterior distribution of $\underline{\omega}$ given data $\underline{X}$ can be written with proportional as follows

$$
\pi(\underline{\omega} \mid \underline{\mathrm{X}}) \propto \pi(\underline{\omega}) \cdot L_{i}(\underline{\omega} \mid \underline{\mathrm{X}}),
$$

hence,

$$
\begin{aligned}
& \pi(\underline{\omega} \mid \underline{\mathrm{X}}) \\
= & \frac{\psi_{1}^{-1}}{\theta \beta}\left(\frac{\alpha \theta}{\beta^{\alpha}}\right)^{V_{i}} \prod_{j=1}^{V_{i}} x_{(j)}^{\alpha} e^{-(U)^{\alpha}}\left(1-e^{-(U)^{\alpha}}\right)^{\theta-1} \\
& \times\left[1-\left(1-e^{-(U)^{\alpha}}\right)^{\theta}\right]^{R_{j}} A_{i}(T ; \underline{\omega}),
\end{aligned}
$$

the normalizing constant $\psi_{1}$ of (13) is given by

$$
\begin{aligned}
\psi_{1} & =\int_{0}^{c} \int_{0}^{\infty} \int_{0}^{\infty} \frac{\alpha^{V_{i}} \theta^{V_{i}-1}}{\beta^{\alpha V_{i}+1}} \cdot \prod_{j=1}^{V_{i}} x_{(j)}^{\alpha} e^{-(U)^{\alpha}}\left(1-e^{-(U)^{\alpha}}\right)^{\theta-1} \\
& \times\left[1-\left(1-e^{-(U)^{\alpha}}\right)^{\theta}\right]^{R_{j}} A_{i}(T ; \underline{\omega}) d \alpha d \beta d \theta,
\end{aligned}
$$

where, $i=1,2,3, \underline{\omega}=(\alpha, \beta, \theta), U=\frac{x_{(j)}}{\beta}$,

$V_{1}=k, \quad A_{1}(T ; \underline{\omega})=1, \quad R_{k}^{*}=n-k-\sum_{i=1}^{k-1} R_{i}$, for Case-I,

$V_{2}=D, \quad A_{2}(T ; \underline{\omega})=\left[1-\left(1-e^{-\left(\frac{T}{\beta}\right)^{\alpha}}\right)^{\theta}\right]^{R_{D+1}^{*}}$,

$R_{D+1}^{*}=n-D-\sum_{i=1}^{D} R_{i}$

for Case-II, 
and

$V_{3}=m, \quad A_{3}(T ; \underline{\omega})=1$,

for Case-III.

Marginal posterior densities of the EW parameters $\alpha, \beta$ and $\theta$ can be obtained, respectively, by integrating (13) with respect to the other two parameters as follows

$$
\begin{array}{cc}
f(\alpha \mid \underline{x})=\psi_{1}^{-1} \alpha^{V_{i}} \prod_{j=1}^{V_{i}} x_{(j)}^{\alpha} M_{1}, & c>\alpha>0, \\
f(\beta \mid \underline{\mathrm{x}})=\left(\psi_{1} \beta\right)^{-1} M_{2}, & \beta>0,
\end{array}
$$

and

$$
f(\theta \mid \underline{\mathrm{x}})=\psi_{1}^{-1} \theta^{V_{i}-1} M_{3}, \quad \theta>0,
$$

where,

$$
\begin{aligned}
M_{1}= & \int_{0}^{\infty} \int_{0}^{\infty} \frac{\theta^{V_{i}-1}}{\beta^{\alpha V_{i}+1}} e^{-(U)^{\alpha}}\left(1-e^{-(U)^{\alpha}}\right)^{\theta-1} \\
& \times\left[1-\left(1-e^{-(U)^{\alpha}}\right)^{\theta}\right]^{R_{j}} A_{i}(T ; \underline{\omega}) d \beta d \theta, \\
M_{2}= & \int_{0}^{c} \int_{0}^{\infty} \frac{\alpha^{V_{i}} \theta^{V_{i}-1}}{\beta^{\alpha V_{i}}} \prod_{j=1}^{V_{i}} x_{(j)}^{\alpha} e^{-(U)^{\alpha}}\left(1-e^{-(U)^{\alpha}}\right)^{\theta-1} \\
& \times\left[1-\left(1-e^{-(U)^{\alpha}}\right)^{\theta}\right]^{R_{j}} A_{i}(T ; \underline{\omega}) d \alpha d \theta,
\end{aligned}
$$

and

$$
\begin{aligned}
M_{3}= & \int_{0}^{c} \int_{0}^{\infty} \frac{\alpha^{V_{i}}}{\beta^{\alpha V_{i}}} \prod_{j=1}^{V_{i}} x_{(j)}^{\alpha} e^{-(U)^{\alpha}}\left(1-e^{-(U)^{\alpha}}\right)^{\theta-1} \\
& \times\left[1-\left(1-e^{-(U)^{\alpha}}\right)^{\theta}\right]^{R_{j}} A_{i}(T ; \underline{\omega}) d \alpha d \beta .
\end{aligned}
$$

Based on the SEL function and the marginal PDF of $\alpha, \beta$ and $\theta$ as in (14), (15) and (16) respectively, the Bayes estimators $\tilde{\alpha}, \tilde{\beta}$ and $\tilde{\theta}$ of the EW parameters $\alpha, \beta$ and $\theta$, respectively, becomes

$$
\begin{gathered}
\tilde{\alpha}=E(\alpha \mid \underline{\mathrm{X}})=\psi_{1}^{-1} \cdot \int_{0}^{c} \alpha^{V_{i}+1} \prod_{j=1}^{V_{i}} x_{(j)}^{\alpha} M_{1} d \alpha, \\
\tilde{\beta}=E(\beta \mid \underline{\mathrm{X}})=\psi_{1}^{-1} \cdot \int_{0}^{\infty} M_{2} d \beta,
\end{gathered}
$$

and

$$
\tilde{\theta}=E(\theta \mid \underline{\mathrm{X}})=\psi_{1}^{-1} \cdot \int_{0}^{\infty} \theta^{V_{i}} M_{3} d \theta
$$

Similarly, based on the marginal PDF of $\alpha, \beta$ and $\theta$ as in (14), (15) and (16) respectively, the corresponding Bayes risk of the Bayes estimators $\tilde{\alpha}, \tilde{\beta}$ and $\tilde{\theta}$ under SEL function will be

$$
R(\tilde{\alpha})=E\left(\alpha^{2} \mid \underline{\mathrm{X}}\right)-[E(\alpha \mid \underline{\mathrm{X}})]^{2},
$$

$$
R(\tilde{\beta})=E\left(\beta^{2} \mid \underline{X}\right)-[E(\beta \mid \underline{X})]^{2},
$$

and

$$
R(\tilde{\theta})=E\left(\theta^{2} \mid \underline{\mathrm{X}}\right)-[E(\theta \mid \underline{\mathrm{X}})]^{2},
$$

where,

$$
\begin{gathered}
E\left(\alpha^{2} \mid \underline{\mathrm{X}}\right)=\psi_{1}^{-1} \cdot \int_{0}^{c} \alpha^{V_{i}+2} \prod_{j=1}^{V_{i}} x_{(j)}^{\alpha} M_{1} d \alpha, \\
E\left(\beta^{2} \mid \underline{\mathrm{X}}\right)=\psi_{1}^{-1} \cdot \int_{0}^{\infty} \beta M_{2} d \beta,
\end{gathered}
$$

and

$$
E\left(\theta^{2} \mid \underline{\mathrm{X}}\right)=\psi_{1}^{-1} \cdot \int_{0}^{\infty} \theta^{V_{i}+2} M_{3} d \theta
$$

Clearly, the Bayes estimators and the corresponding Bayes risks of the EW parameters $\alpha, \beta$ and $\theta$ based on Type-I GPHCS do not result in closed forms due to involvement of multidimensional integrals which are not solvable analytically. Therefore, the Bayes estimates and the Bayes risks of $\tilde{\alpha}, \tilde{\beta}$ and $\tilde{\theta}$ can be evaluated by using computer facilities and numerical techniques.

\subsection{Bayes Estimators of EW Parameters Based on Type-II GPHCS}

Based on the likelihood function (8), non-informative priors (12) and using the Bayes theorem, the joint posterior distribution of $\underline{\omega}$ given data $\underline{X}$ can be written with proportional as follows

$$
\pi(\underline{\omega} \mid \underline{\mathrm{X}}) \propto \pi(\underline{\omega}) \cdot L_{i}(\underline{\omega} \mid \underline{\mathrm{X}}),
$$

hence,

$$
\begin{aligned}
& \pi(\underline{\omega} \mid \underline{\mathrm{X}}) \\
= & \frac{\psi_{2}^{-1}}{\theta \beta}\left(\frac{\alpha \theta}{\beta^{\alpha}}\right)^{S_{i}} \prod_{j=1}^{S_{i}} x_{(j)}^{\alpha} e^{-(U)^{\alpha}}\left(1-e^{-(U)^{\alpha}}\right)^{\theta-1} \\
& \times\left[1-\left(1-e^{-(U)^{\alpha}}\right)^{\theta}\right]^{R_{j}} A_{i}\left(T_{\tau} ; \underline{\omega}\right),
\end{aligned}
$$

the normalizing constant $\psi_{2}$ of (17) is given by

$$
\begin{aligned}
\psi_{2} & =\int_{0}^{c} \int_{0}^{\infty} \int_{0}^{\infty} \frac{\alpha^{S_{i}} \theta^{S_{i}-1}}{\beta^{\alpha S_{i}+1}} \prod_{j=1}^{S_{i}} x_{(j)}^{\alpha} e^{-(U)^{\alpha}}\left(1-e^{-(U)^{\alpha}}\right)^{\theta-1} \\
& \times\left[1-\left(1-e^{-(U)^{\alpha}}\right)^{\theta}\right]^{R_{j}} A_{i}\left(T_{\tau} ; \underline{\omega}\right) d \alpha d \beta d \theta,
\end{aligned}
$$

where, $i=1,2,3, \quad \underline{\omega}=(\alpha, \beta, \theta), \quad U=\frac{x_{(j)}}{\beta}, \tau=1,2$, for $i=1,3$, respectively, 
$S_{1}=D_{1}, \quad A_{1}\left(T_{1} ; \underline{\omega}\right)=\left[1-\left(1-e^{-\left(\frac{T_{1}}{\beta}\right)^{\alpha}}\right)^{\theta}\right]^{R_{D_{1}+1}^{*}}$

$R_{D_{1}+1}^{*}=n-d_{1}-\sum_{i=1}^{m-1} R_{i}$,

for Case-I,

$S_{2}=m, \quad A_{2}\left(T_{\tau} ; \underline{\omega}\right)=1$,

for Case-II,

and

$S_{3}=D_{2}, A_{3}\left(T_{2} ; \underline{\omega}\right)=\left[1-\left(1-e^{-\left(\frac{T_{2}}{\beta}\right)^{\alpha}}\right)^{\theta}\right]^{R_{D_{2}+1}^{*}}$,

$R_{D_{2}+1}^{*}=n-d_{2}-\sum_{i=1}^{d_{2}} R_{i}$,

for Case-III.

Marginal posterior densities of $\alpha, \beta$ and $\theta$ obtained by integrating (17) with respect to the other two parameters as follows

$$
\begin{gathered}
f(\alpha \mid \underline{x})=\psi_{2}^{-1} \alpha S_{i} \prod_{j=1}^{S_{i}} x_{(j)}^{\alpha} M_{4}, \quad c>\alpha>0, \\
f(\beta \mid \underline{\mathrm{x}})=\left(\psi_{2} \beta\right)^{-1} M_{5}, \quad \beta>0,
\end{gathered}
$$

and

$$
f(\theta \mid \underline{\mathbf{x}})=\psi_{2}^{-1} \theta^{S_{i}-1} M_{6}, \quad \theta>0,
$$

where,

$$
\begin{aligned}
M_{4}= & \int_{0}^{\infty} \int_{0}^{\infty} \frac{\theta^{S_{i}-1}}{\beta^{\alpha S_{i}+1}} \cdot e^{-(U)^{\alpha}}\left(1-e^{-(U)^{\alpha}}\right)^{\theta-1} \\
& \times\left[1-\left(1-e^{-(U)^{\alpha}}\right)^{\theta}\right]^{R_{j}} A_{i}\left(T_{\tau} ; \underline{\omega}\right) d \beta d \theta, \\
M_{5}= & \int_{0}^{c} \int_{0}^{\infty} \frac{\alpha^{S_{i}} \theta^{S_{i}-1}}{\beta^{\alpha S_{i}}} \prod_{j=1}^{S_{i}} x_{(j)}^{\alpha} e^{-(U)^{\alpha}}\left(1-e^{-(U)^{\alpha}}\right)^{\theta-1} \\
& \times\left[1-\left(1-e^{-(U)^{\alpha}}\right)^{\theta}\right]^{R_{j}} A_{i}\left(T_{\tau} ; \underline{\omega}\right) d \alpha d \theta,
\end{aligned}
$$

and

$$
\begin{aligned}
M_{6}=\int_{0}^{c} \int_{0}^{\infty} \frac{\alpha^{S_{i}}}{\beta^{\alpha S_{i}}} \prod_{j=1}^{S_{i}} x_{(j)}^{\alpha} e^{-(U)^{\alpha}}\left(1-e^{-(U)^{\alpha}}\right)^{\theta-1} \\
\times\left[1-\left(1-e^{-(U)^{\alpha}}\right)^{\theta}\right]^{R_{j}} A_{i}\left(T_{\tau} ; \underline{\omega}\right) d \alpha d \beta .
\end{aligned}
$$

Based on the SEL function and the marginal PDF of $\alpha, \beta$ and $\theta$ as in (18), (19) and (20) respectively, the Bayes estimators $\tilde{\alpha}, \tilde{\beta}$ and $\tilde{\theta}$ of the EW parameters $\alpha, \beta$ and $\theta$ can be defined, respectively, as follows

$$
\begin{gathered}
\tilde{\alpha}=E(\alpha \mid \underline{\mathrm{X}})=\psi_{2}^{-1} \cdot \int_{0}^{c} \alpha^{S_{i}+1} \prod_{j=1}^{V_{i}} x_{(j)}^{\alpha} M_{4} d \alpha, \\
\tilde{\beta}=E(\beta \mid \underline{\mathrm{X}})=\psi_{2}^{-1} \cdot \int_{0}^{\infty} M_{5} d \beta,
\end{gathered}
$$

and

$$
\tilde{\theta}=E(\theta \mid \underline{\mathrm{X}})=\psi_{2}^{-1} \cdot \int_{0}^{\infty} \theta^{S_{i}} M_{6} d \theta .
$$

Similarly, based on the marginal PDF of $\alpha, \beta$ and $\theta$ as in (18), (19) and (20) respectively, the Bayes risk associated with $\tilde{\alpha}, \tilde{\beta}$ and $\tilde{\theta}$ under SEL function will be

$$
\begin{aligned}
& R(\tilde{\alpha})=E\left(\alpha^{2} \mid \underline{\mathrm{X}}\right)-[E(\alpha \mid \underline{\mathrm{X}})]^{2}, \\
& R(\tilde{\beta})=E\left(\beta^{2} \mid \underline{\mathrm{X}}\right)-[E(\beta \mid \underline{\mathrm{X}})]^{2},
\end{aligned}
$$

and

$$
R(\tilde{\theta})=E\left(\theta^{2} \mid \underline{\mathrm{X}}\right)-[E(\theta \mid \underline{\mathrm{x}})]^{2},
$$

where,

$$
\begin{gathered}
E\left(\alpha^{2} \mid \underline{\mathrm{X}}\right)=\psi_{2}^{-1} \cdot \int_{0}^{c} \alpha^{S_{i}+2} \prod_{j=1}^{S_{i}} x_{(j)}^{\alpha} M_{4} d \alpha, \\
E\left(\beta^{2} \mid \underline{\mathrm{X}}\right)=\psi_{2}^{-1} \cdot \int_{0}^{\infty} \beta M_{5} d \beta,
\end{gathered}
$$

and

$$
E\left(\theta^{2} \mid \underline{\mathrm{X}}\right)=\psi_{2}^{-1} \cdot \int_{0}^{\infty} \theta^{S_{i}+2} M_{6} d \theta
$$

Again, computer facilities and numerical techniques are needed to solving this set of nonlinear equations due to the Bayes estimators and Bayes risks of the EW parameters $\alpha, \beta$ and $\theta$ based on Type-II GPHCS do not result in closed forms.

\section{Real Data Analysis}

Previous sections dealt with the analytical technique and this section focuses on the numerical one through practical data set, which was originally presented by Nichols and Padgett [13]. This data set was obtained from a process producing carbon fibers to be used in constructing fibrous composite materials. The ordered data with $n=100$ observations on breaking stress of carbon fibers (in Gba) are in Table 1.

Table 1. Breaking stress of carbon fibers

\begin{tabular}{|llllllllll|}
\hline 0.39 & 0.81 & 0.85 & 0.98 & 1.08 & 1.12 & 1.17 & 1.18 & 1.22 & 1.25 \\
1.36 & 1.41 & 1.47 & 1.57 & 1.57 & 1.59 & 1.59 & 1.61 & 1.61 & 1.69 \\
1.69 & 1.71 & 1.73 & 1.80 & 1.84 & 1.84 & 1.87 & 1.89 & 1.92 & 2.00 \\
2.03 & 2.03 & 2.05 & 2.12 & 2.17 & 2.17 & 2.17 & 2.35 & 2.38 & 2.41 \\
2.43 & 2.48 & 2.48 & 2.50 & 2.53 & 2.55 & 2.55 & 2.56 & 2.59 & 2.67 \\
2.73 & 2.74 & 2.76 & 2.77 & 2.79 & 2.81 & 2.81 & 2.82 & 2.83 & 2.85 \\
2.87 & 2.88 & 2.93 & 2.95 & 2.96 & 2.97 & 2.97 & 3.09 & 3.11 & 3.11 \\
3.15 & 3.15 & 3.19 & 3.19 & 3.22 & 3.22 & 3.27 & 3.28 & 3.31 & 3.31 \\
3.33 & 3.39 & 3.39 & 3.51 & 3.56 & 3.60 & 3.65 & 3.68 & 3.68 & 3.68 \\
3.70 & 3.75 & 4.20 & 4.38 & 4.42 & 4.70 & 4.90 & 4.91 & 5.08 & 5.56 \\
\hline
\end{tabular}


One question arises about whether the data fit the EW distribution or not. To check for the goodness of fit, we compute the chi-square test. First, the EW distribution will be fitting using the MLEs and then carrying out chi-square goodness of fit test. The MLEs $\underline{\hat{\omega}}=(\hat{\alpha}, \hat{\beta}, \hat{\theta})$ for the unknown parameters $\underline{\omega}=(\alpha, \beta, \theta)$ of the EW distribution, respectively, will be

$$
\begin{aligned}
\hat{\alpha}(\hat{\beta}, \hat{\theta}) & n \cdot\left\{n \log \hat{\beta}-\sum_{i=1}^{n} \log \left(x_{i}\right)+\sum_{i=1}^{n} \mathfrak{J}^{\hat{\alpha}} \log \mathfrak{I}\right. \\
& \left.-(\hat{\theta}-1) \cdot \sum_{i=1}^{n} e^{-(\mathfrak{I})^{\hat{\alpha}}} \mathfrak{J}^{\hat{\alpha}} \log (\mathfrak{I})\left(1-e^{-(\mathfrak{I})^{\hat{\alpha}}}\right)^{-1}\right\}^{-1},
\end{aligned}
$$$$
\hat{\beta}(\hat{\alpha}, \hat{\theta})
$$$$
=-n \hat{\alpha} \cdot\left\{\begin{array}{l}
\frac{\hat{\alpha}(\hat{\theta}-1)}{\hat{\beta}} \cdot \sum_{i=1}^{n} e^{-(\mathfrak{I})^{\hat{\alpha}}}(\mathfrak{I})^{\hat{\alpha}}\left(1-e^{-(\mathfrak{I})^{\hat{\alpha}}}\right)^{-1} \\
-\frac{\hat{\alpha}}{\hat{\beta}} \cdot \sum_{i=1}^{n}(\mathfrak{I})^{\hat{\alpha}}
\end{array}\right\}
$$

and

$$
\hat{\theta}(\hat{\alpha}, \hat{\beta})=n \cdot\left\{-\sum_{i=1}^{n} \log \left(1-e^{-(\Im) \hat{\alpha}}\right)\right\}^{-1}
$$

where, $\mathfrak{I}=\frac{x_{i}}{\hat{\beta}}$.

Using the MathCad package and the real data set as in Table 1, the maximum likelihood estimates of the unknown EW parameters $\alpha, \beta$ and $\theta$ will be $\hat{\alpha}=2.438$, $\hat{\beta}=2.702$ and $\hat{\theta}=1.294$. The chi-square goodness of fit test is a hypothesis test. The null and alternative hypotheses being tested are:

$H_{0}$ : The data set follow the EW distribution.

$H_{1}$ : The data set do not follow the EW distribution.

For chi-square goodness of fit test, the data are divided into $k$ bins and the test statistic is defined as

$$
\chi_{c}^{2}=\sum_{i=1}^{k}\left(O_{i}-E_{i}\right)^{2} / E_{i},
$$

where, $O_{i}$ and $E_{i}$, is the observed and expected frequency for bin $i$. The expected frequency is calculated by

$$
E_{i}=n \cdot\left[F\left(y_{U}\right)-F\left(y_{L}\right)\right]
$$

where, $F$ is the CDF of the EW distribution, $Y_{U}$ and $Y_{L}$ are the lower and upper limits for class $i$ and $n$ is the sample size. The observed and the expected frequencies of the ordered data set can be calculated and reported in Table 2:

Table 2. Computed the expected frequencies of the data set

\begin{tabular}{|c|c|c|c|}
\hline$i$ & $O_{i}$ & $E_{i}$ & $\left(O_{i}-E_{i}\right)^{2} / E_{i}$ \\
\hline$(0,1.5]$ & 13 & 13.42 & 0.013 \\
$(1.5,2]$ & 17 & 15.31 & 0.187 \\
$(2,2.5]$ & 14 & 18.81 & 1.230 \\
$(2.5,3]$ & 23 & 18.41 & 1.144 \\
$(3,6]$ & 33 & 33.93 & 0.025 \\
\hline \multicolumn{3}{|c|}{ Sum } & $\mathbf{2 . 5 9 9}$ \\
\hline
\end{tabular}

Since $\chi_{\mathrm{C}}^{2}=2.599$ less than the tabulated value $\chi_{1,0.05}^{2}=3.84$, we cannot reject the null hypothesis that the data are coming from the EW distribution at significance level 0.05 . Now, we created an artificial data by progressive Type-II censoring, we have $n=100$ and $m=40$, at the time of any observed failure $R_{j}=1$ of the survival items will be withdrawn from the life test at random. Then, the observed failures of Type-II progressive censored sample are: $0.39,0.85,0.98,1.12$, $1.17,1.18,1.22,1.36,1.41,1.57,1.57,1.59,1.61,1.61$, $1.69,1.69,1.71,1.73,1.80,1.84,1.84,1.87,1.92,2.03$, $2.03,2.20,2.17,2.17,2.17,2.35,2.38,2.41,2.48,2.48$, 2.50, 2.53, 2.55, 2.55, 2.56, 2.59 .

To obtain the maximum likelihood estimates and Bayes estimates for the EW parameters under Type-I and Type-II GPHCSs, the progressively Type-II censored sample will be proposed in a design under Type-I and Type-II GPHCSs as in Table 3.

Notice that, in Table 3, (-) represents to a number of observed failures at time which is less than the termination point of the life test.

All computations were performed using MathCad package version 14 . The maximum likelihood estimates and approximate CIs for the unknown parameters of EW distribution $\alpha, \beta$ and $\theta$ based on Type-I and Type-II GPHCSs are calculated and reported in Table 4 and Table 5 , respectively. To evaluate the Bayes estimators, some various values of hyper-parameter $c=1,2$ and 4 are considered. The Bayes estimates and the corresponding Bayes risks of the unknown EW parameters based on Type-I and Type-II GPHCSs are reported in Table 6 and Table 7 , respectively.

Table 3. Design the progressively Type-II censored sample of EW distribution based on Type-I and Type-II GPHCSs

\begin{tabular}{|l|c|c|c|c|c|c|c|c|c|c|}
\hline \multirow{2}{*}{ Scheme } & \multirow{2}{*}{$n$} & \multicolumn{4}{|c|}{ Type-I GPHCS } & \multicolumn{5}{c|}{ Type-II GPHCS } \\
\cline { 3 - 11 } & & $d$ & $T$ & $k$ & $m$ & $d_{1}$ & $T_{1}$ & $d_{2}$ & $T_{2}$ & $m$ \\
\hline Case-I & 100 & 25 & 1.5 & 30 & 40 & 40 & 3.2 & - & - & 35 \\
Case-II & 100 & 35 & 2.5 & 30 & 40 & 30 & 2 & - & - & 40 \\
Case-III & 100 & - & - & 35 & 40 & 30 & 2 & 35 & 2.5 & 40 \\
\hline
\end{tabular}




\section{Conclusions}

In this paper, we have considered the Bayes and nonBayes estimations for the unknown parameters of the EW distribution based on Type-I and Type-II GPHCSs. Some special cases using exponential and Weibull distributions are obtained, i.e., based on Type-I GPHCS, Cho et al. [4] results were generalized in the case of exponential and Weibull distributions at $\alpha=\theta=1$ and $\theta=1$, respectively. Also, based on Type-II GPHCS, Lee et al. [7] results in the case of exponential parameter can be obtained as a special case at $\alpha=\theta=1$ as well as if putting $\theta=1$, Ashour and Elshahhat [1] results can be obtained as a special case in the case of Weibull distribution. The MLEs, approximate V-Cov matrix and the approximate CIs based on the observed Fisher information matrix have been discussed. Independent non-informative priors are considered to provide the Bayes estimators and the corresponding Bayes risks under the SEL function. Based on Type-I and Type-II GPHCSs, the MLEs and the Bayes estimators for EW parameters do not result in explicit forms, therefore, a numerical example has been presented to illustrate all the inferential results established here. As expected, Table 4 and Table 5 showed that the maximum likelihood estimates for the unknown parameters of EW distribution based on both Types of GPHCSs are more precise than the Bayes estimates as in Table 6 and Table 7. Therefore, if prior information of the EW parameters is not available, then it is always better to use the MLEs rather than the Bayes estimators, because the Bayes estimators are computationally more expensive.

Table 4. The maximum likelihood estimates, corresponding variances and approximate $95 \%$ two-sided CIs for EW parameters under Type-I GPHCS

\begin{tabular}{|c|c|c|c|c|c|c|c|c|c|c|}
\hline \multirow{2}{*}{ Scheme } & \multicolumn{9}{|c|}{ Type-I GPHCS } \\
\cline { 2 - 11 } & \multicolumn{3}{|c|}{ Estimates } & \multicolumn{3}{c|}{ Variances } & \multicolumn{3}{c|}{ CI } \\
\cline { 2 - 10 } & $\hat{\alpha}$ & $\hat{\beta}$ & $\hat{\theta}$ & $\hat{\sigma}_{\alpha}^{2}$ & $\hat{\sigma}_{\beta}^{2}$ & $\hat{\sigma}_{\theta}^{2}$ & $\alpha$ & \multicolumn{2}{c|}{$\beta$} & $\theta$ \\
\hline Case-I & 1.872 & 2.487 & 1.895 & 0.093 & 0.098 & 0.091 & $(1.27,2.46)$ & $(1.87,3.10)$ & $(1.30,2.48)$ \\
Case-II & 1.849 & 2.512 & 1.975 & 0.080 & 0.026 & 0.067 & $(1.29,2.40)$ & $(2.19,2.82)$ & $(1.47,2.48)$ \\
Case-III & 2.446 & 2.019 & 2.025 & 0.119 & 0.007 & 0.075 & $(1.76,3.12)$ & $(1.85,2.18)$ & $(1.48,2.56)$ \\
\hline
\end{tabular}

Table 5. The maximum likelihood estimates, corresponding variances and approximate $95 \%$ two-sided CIs for EW parameters under Type-II GPHCS

\begin{tabular}{|c|c|c|c|c|c|c|c|c|c|}
\hline \multirow{3}{*}{ Scheme } & \multicolumn{9}{|c|}{ Type-II GPHCS } \\
\hline & \multicolumn{3}{|c|}{ Estimates } & \multicolumn{3}{|c|}{ Variances } & \multicolumn{3}{|c|}{ CI } \\
\hline & $\hat{\alpha}$ & $\hat{\beta}$ & $\hat{\theta}$ & $\hat{\sigma}_{\alpha}^{2}$ & $\hat{\sigma}_{\beta}^{2}$ & $\hat{\sigma}_{\theta}^{2}$ & $\alpha$ & $\beta$ & $\theta$ \\
\hline Case-I & 1.685 & 2.730 & 1.750 & 0.057 & 0.033 & 0.049 & $(1.22,2.15)$ & $(2.37,3.08)$ & $(1.32,2.18)$ \\
\hline Case-II & 2.287 & 1.946 & 2.250 & 0.102 & 0.007 & 0.094 & $(1.66,2.91)$ & $(1.78,2.11)$ & $(1.65,2.85)$ \\
\hline Case-III & 1.921 & 2.391 & 1.975 & 0.087 & 0.018 & 0.066 & $(1.34,2.50)$ & $(2.12,2.65)$ & $(1.47,2.48)$ \\
\hline
\end{tabular}

Table 6. The Bayes estimates and corresponding Bayes risks for EW parameters under Type-I GPHCS

\begin{tabular}{|c|c|c|c|c|c|c|c|}
\hline \multirow{3}{*}{$\begin{array}{c}\text { Hyper } \\
\text { Parameter }\end{array}$} & \multirow{3}{*}{ Scheme } & \multicolumn{6}{|c|}{ Type-I GPHCS } \\
\hline & & \multicolumn{3}{|c|}{ Bayes Estimates } & \multicolumn{3}{|c|}{ Variances } \\
\hline & & $\tilde{\alpha}$ & $\tilde{\beta}$ & $\tilde{\theta}$ & $\operatorname{Var}(\tilde{\alpha})$ & $\operatorname{Var}(\tilde{\beta})$ & $\operatorname{Var}(\tilde{\theta})$ \\
\hline \multirow{3}{*}{$c=1$} & Case-I & 0.722 & 0.802 & 12.336 & 0.027 & 0.207 & 61.337 \\
\hline & Case-II & 0.746 & 0.774 & 12.846 & 0.023 & 0.164 & 63.259 \\
\hline & Case-III & 0.872 & 0.654 & 13.780 & 0.011 & 0.021 & 39.009 \\
\hline \multirow{3}{*}{$c=2$} & Case-I & 1.285 & 1.746 & 5.869 & 0.226 & 0.714 & 40.350 \\
\hline & Case-II & 1.422 & 1.819 & 4.894 & 0.252 & 0.477 & 31.296 \\
\hline & Case-III & 1.456 & 1.418 & 5.034 & 0.064 & 0.132 & 10.103 \\
\hline \multirow{3}{*}{$c=4$} & Case-I & 2.263 & 2.435 & 3.532 & 1.334 & 0.918 & 32.317 \\
\hline & Case-II & 2.565 & 2.544 & 2.747 & 1.274 & 0.675 & 22.584 \\
\hline & Case-III & 2.648 & 2.131 & 1.688 & 0.166 & 0.055 & 2.031 \\
\hline
\end{tabular}


Table 7. The Bayes estimates and corresponding Bayes risks for EW parameters under Type-II GPHCS

\begin{tabular}{|c|c|c|c|c|c|c|c|}
\hline \multirow{3}{*}{$\begin{array}{c}\text { Hyper } \\
\text { Parameter }\end{array}$} & \multirow{3}{*}{ Scheme } & \multicolumn{6}{|c|}{ Type-II GPHCS } \\
\hline & & \multicolumn{3}{|c|}{ Bayes Estimates } & \multicolumn{3}{|c|}{ Variances } \\
\hline & & $\tilde{\alpha}$ & $\tilde{\beta}$ & $\tilde{\theta}$ & $\operatorname{Var}(\tilde{\alpha})$ & $\operatorname{Var}(\tilde{\beta})$ & $\operatorname{Var}(\tilde{\theta})$ \\
\hline \multirow{3}{*}{$c=1$} & Case-I & 0.739 & 0.818 & 9.669 & 0.021 & 0.170 & 20.261 \\
\hline & Case-II & 0.888 & 0.698 & 12.008 & 0.005 & 0.018 & 7.856 \\
\hline & Case-III & 0.803 & 0.893 & 9.442 & 0.018 & 0.108 & 15.792 \\
\hline \multirow{3}{*}{$c=2$} & Case-I & 0.999 & 1.443 & 6.357 & 0.142 & 0.735 & 23.363 \\
\hline & Case-II & 1.641 & 1.562 & 3.976 & 0.067 & 0.078 & 3.705 \\
\hline & Case-III & 1.374 & 1.820 & 4.423 & 0.162 & 0.434 & 12.483 \\
\hline \multirow{3}{*}{$c=4$} & Case-I & 1.287 & 1.792 & 5.812 & 0.559 & 1.191 & 23.761 \\
\hline & Case-II & 2.943 & 2.193 & 1.653 & 0.340 & 0.069 & 0.841 \\
\hline & Case-III & 2.395 & 2.521 & 2.429 & 0.886 & 0.571 & 8.619 \\
\hline
\end{tabular}

\section{References}

[1] Ashour, S. \& Elshahhat, A. (2016). Bayesian and non-Bayesian estimation for Weibull parameters based on generalized Type-II progressive hybrid censoring scheme. Pakistan Journal of Statistics \& Operation Research, 12(2), 213-226.

[2] Childs, A., Chandrasekar, B. \& Balakrishnan, N. (2008). Exact likelihood inference for an exponential parameter under progressive hybrid censoring schemes. In Statistical Models and Methods for Biomedical and Technical Systems, Vonta, F., Nikulin, M., Limnios, N. \& Huber-Carol, C, (Eds), Birkhäuser, Boston, 319-330.

[3] Cho, Y., Sun, H., \& Lee, K. (2015a). Estimating the entropy of a Weibull distribution under generalized progressive hybrid censoring. Entropy, 17(1), 102-122.

[4] Cho, Y., Sun, H., \& Lee, K. (2015b). Exact likelihood inference for an exponential parameter under generalized progressive hybrid censoring scheme. Statistical Methodology, 23, 18-34.

[5] Cohen, A. C. (1965). Maximum likelihood estimation in the Weibull distribution based on complete and censored samples. Technometrics, 7(4), 579-588.

[6] Kundu, D. \& Joarder, A. (2006). Analysis of Type-II progressively hybrid censored data. Computational Statistics \& Data Analysis, 50(10), 2509-2528.

[7] Lee, K., Sun, H., \& Cho, Y. (2016a). Exact likelihood inference of the exponential parameter under generalized Type-II progressive hybrid censoring. Journal of the Korean Statistical Society, 45(1), 123-136.

[8] Lee, K. J., Lee, J. I., \& Park, C. K. (2016b). Analysis of generalized progressive hybrid censored competing risks data. Journal of the Korean Society of Marine Engineering, 40(2), 131137.

[9] Mudholkar, G. S., \& Srivastava, D. K. (1993). Exponentiated Weibull family for analyzing bathtub failure-rate data. IEEE Transactions on Reliability, 42(2), 299-302.

[10] Mudholkar, G. S., Srivastava, D. K., \& Freimer, M. (1995). The exponentiated Weibull family: a reanalysis of the bus-motorfailure data. Technometrics, 37(4), 436-445.

[11] Mudholkar, G. S., \& Hutson, A. D. (1996). The exponentiated Weibull family: some properties and a flood data application. Communications in Statistics-Theory \& Methods, 25(12), 30593083.

[12] Nassar, M. M., \& Eissa, F. H. (2003). On the exponentiated Weibull distribution. Communications in Statistics-Theory \& Methods, 32(7), 1317-1336.

[13] Nichols, M. D., \& Padgett, W. J. (2006). A bootstrap control chart for Weibull percentiles. Quality \& Reliability Engineering International, 22(2), 141-151.

[14] Singh, U., Gupta, P. K., \& Upadhyay, S. K. (2005). Estimation of three-parameter exponentiated-Weibull distribution under Type-II censoring. Journal of Statistical Planning \& Inference, 134(2), 350-372.

\section{Appendix A:}

The elements of the observed Fisher information matrix (11) based on the log-likelihood function (6) will be 


$$
\begin{aligned}
& \frac{\partial^{2} l_{i}(\underline{\omega} \mid \underline{X})}{\partial \alpha^{2}}=-\frac{V_{i}}{\alpha^{2}}-\sum_{j=1}^{V_{i}} U^{\alpha} \log U^{2}-(\theta-1) \sum_{j=1}^{V_{i}} e^{-(U)^{\alpha}}\left\{U^{\alpha} \log U\right\}^{2} \xi_{U}^{-1} \\
& \times\left\{1-U^{-\alpha}+e^{-(U)^{\alpha}} \xi_{U}^{-1}\right\}-\theta \sum_{j=1}^{V_{i}} R_{j} e^{-(U)^{\alpha}} U^{\alpha} \log U^{2} \xi_{U}^{\theta-1}\left\{1-\xi_{U}^{\theta}\right\}^{-1} \\
& \times\left\{1-U^{\alpha}+(\theta-1) e^{-(U)^{\alpha}} U^{\alpha} \xi_{U}^{-1}+\theta e^{-(U)^{\alpha}} U^{\alpha} \xi_{U}^{\theta-1}\left\{1-\xi_{U}^{\theta}\right\}^{-1}\right\} \\
& -\theta R_{D+1}^{*}\left\{\theta e^{-2(Q)^{\alpha}}\left\{Q^{\alpha} \log Q\right\}^{2} \xi_{Q}^{2(\theta-1)}\left\{1-\xi_{Q}^{\theta}\right\}^{-2}+\left\{1-\xi_{Q}^{\theta}\right\}^{-1}\right. \\
& \left.\times\left\{e^{-(Q)^{\alpha}} \xi_{Q}^{\theta-1} Q^{\alpha} \log Q^{2}\left[1-Q^{\alpha}+(\theta-1) e^{-(Q)^{\alpha}} Q^{\alpha} \xi_{Q}^{-1}\right]\right\}\right\} . \\
& \frac{\partial^{2} l_{i}(\underline{\omega} \mid \underline{\mathrm{X}})}{\partial \beta^{2}}=\frac{\alpha V_{i}}{\beta^{2}}-\frac{\alpha(1+\alpha)}{\beta^{2}} \sum_{j=1}^{V_{i}} U^{\alpha}+\frac{\alpha(\theta-1)}{\beta^{2}} \sum_{j=1}^{V_{i}} e^{-(U)^{\alpha}} U^{\alpha} \xi_{U}^{-1} \\
& \times\left\{1+\alpha-U-e^{-(U)^{\alpha}} U^{\alpha} \xi_{U}^{-1}\right\}-\frac{\alpha \theta}{\beta} \sum_{j=1}^{V_{i}} R_{j} e^{-(U)^{\alpha}} U^{\alpha} \xi_{U}^{\theta-1}\left\{1-\xi_{U}^{\theta}\right\}^{-1} \\
& \times\left\{\frac{1}{\beta}+\frac{\alpha}{\beta}-\alpha U^{\alpha}+\frac{\alpha(\theta-1)}{\beta} e^{-(U)^{\alpha}} U^{\alpha} \xi_{U}^{-1}+\frac{\alpha \theta}{\beta} e^{-(U)^{\alpha}} U^{\alpha} \xi_{U}^{\theta-1}\left\{1-\xi_{U}^{\theta}\right\}^{-1}\right\} \\
& +\frac{\alpha \theta}{\beta}\left\{-\frac{\alpha \theta}{\beta^{2}} R_{D+1}^{*} e^{-2(Q)^{\alpha}} Q^{2 \alpha} \xi_{Q}^{2(\theta-1)}\left\{1-\xi_{Q}^{\theta}\right\}^{-2}+\left\{1-\xi_{Q}^{\theta}\right\}^{-1}\left\{-\frac{\alpha(\theta-1)}{\beta}\right.\right. \\
& \left.\left.\times e^{-2(Q)^{\alpha}} Q^{2 \alpha} \xi_{Q}^{\theta-2}+\xi_{Q}^{\theta-1}\left\{\frac{\alpha}{\beta} R_{D+1}^{*} e^{-(Q)^{\alpha}} Q^{2 \alpha}\left[1-(Q)^{-\alpha}\right]\right\}\right\}\right\}-\frac{\alpha \theta}{\beta^{2}} R_{D+1}^{*} \\
& \times e^{-(Q)^{\alpha}} Q^{\alpha} \xi_{Q}^{\theta-1}\left[1-\xi_{Q}^{\theta}\right]^{-1} \text {. }
\end{aligned}
$$$$
\frac{\partial^{2} l_{i}(\underline{\omega} \mid \underline{X})}{\partial \theta^{2}}=-\frac{V_{i}}{\theta^{2}}-\left\{\sum_{j=1}^{V_{i}} R_{j} \xi_{U}^{\theta} \log \xi_{U}^{2}\left\{1-\xi_{U}^{\theta}\right\}^{-1}\left[1+\xi_{U}^{\theta}\left\{1-\xi_{U}^{\theta}\right\}^{-1}\right]\right\}
$$$$
-R_{D+1}^{*} \xi_{Q}^{\theta} \log \xi_{Q}^{2}\left\{1-\xi_{Q}^{\theta}\right\}^{-1}\left\{1+\xi_{Q}^{\theta}\left\{1-\xi_{Q}^{\theta}\right\}^{-1}\right\} \text {. }
$$

$$
\begin{aligned}
\frac{\partial^{2} l_{i}(\underline{\omega} \mid \underline{X})}{\partial \alpha \partial \theta} & =\sum_{j=1}^{V_{i}} e^{-(U)^{\alpha}} U^{\alpha} \log U \xi_{U}^{-1}-\sum_{j=1}^{V_{i}} R_{j} e^{-(U)^{\alpha}} U^{\alpha} \log U \xi_{U}^{\theta-1}\left\{1-\xi_{U}^{\theta}\right\}^{-1} \\
& \times\left\{1+\theta \log \xi_{U}+\theta \xi_{U}^{\theta} \log \xi_{U}\left\{1-\xi_{U}^{\theta}\right\}^{-1}\right\}-R_{D+1}^{*} e^{-(Q)^{\alpha}} Q^{\alpha} \log Q \xi_{Q}^{\theta-1}\left\{1-\xi_{Q}^{\theta}\right\}^{-1} \\
& \times\left\{1+\theta \log \xi_{Q}+\theta \xi_{Q}^{\theta} \log \xi_{Q}\left\{1-\xi_{Q}^{\theta}\right\}^{-1}\right\}
\end{aligned}
$$




$$
\begin{aligned}
\frac{\partial^{2} l_{i}(\underline{\underline{\omega}} \mid \underline{\mathrm{X}})}{\partial \beta \partial \alpha} & =-\frac{V_{i}}{\beta}+\frac{1}{\beta} \sum_{j=1}^{V_{i}} U^{\alpha}\{1+\alpha \log U\}-\frac{(\theta-1)}{\beta} \sum_{j=1}^{V_{i}} e^{-(U)^{\alpha}} U^{\alpha} \xi_{U}^{-1} \\
& \times\left\{1+\alpha \log U\left[1-U^{\alpha}-e^{-(U)^{\alpha}} U^{\alpha} \xi_{U}^{-1}\right]\right\}+\frac{\theta}{\beta} \sum_{j=1}^{V_{i}} R_{j} e^{-(U)^{\alpha}} U^{\alpha} \xi_{U}^{\theta-1}\left\{1-\xi_{U}^{\theta}\right\}^{-1} \\
& \times\left\{1+\alpha \log U\left[1-U^{\alpha}+(\theta-1) e^{-(U)^{\alpha}} U^{\alpha} \xi_{U}^{-1}+\theta e^{-(U)^{\alpha}} U^{\alpha} \xi_{U}^{\theta-1}\left\{1-\xi_{U}^{\theta}\right\}^{-1}\right]\right\} \\
& +\frac{\alpha \theta^{2}}{\beta} R_{D+1}^{*} e^{-2(Q)^{\alpha}} Q^{2 \alpha} \log Q \xi_{Q}^{2(\theta-1)}\left[1-\xi_{Q}^{\theta}\right]^{-2}+\left[1-\xi_{Q}^{\theta}\right]^{-1}\left\{\frac{\alpha \theta(\theta-1)}{\beta} R_{D+1}^{*}\right. \\
& \times e^{-2(Q)^{\alpha}} Q^{2 \alpha} \log Q \xi_{Q}^{\theta-2}+\frac{\theta}{\beta} R_{D+1}^{*} e^{-(Q)^{\alpha}} Q^{\alpha} \xi_{Q}^{\theta-1}+\frac{\theta}{\beta} R_{D+1}^{*} e^{-(Q)^{\alpha}} Q^{\alpha} \xi_{Q}^{\theta-1} \\
& \left.\times\left[1+\alpha \log Q\left\{1-Q^{\alpha}\right\}\right]\right\} .
\end{aligned}
$$

and

$$
\begin{aligned}
\frac{\partial^{2} l_{i}(\underline{\omega} \mid \underline{X})}{\partial \beta \partial \theta} & =-\frac{\alpha}{\beta} \sum_{j=1}^{V_{i}} e^{-(U)^{\alpha}} U^{\alpha} \xi_{U}^{-1}+\frac{\alpha}{\beta} \sum_{j=1}^{V_{i}} R_{j} e^{-(U)^{\alpha}} U^{\alpha} \xi_{U}^{\theta-1}\left\{1-\xi_{U}^{\theta}\right\}^{-1} \\
& \times\left\{1+\theta \log \xi_{U}\left[1+\xi_{U}^{\theta}\left\{1-\xi_{U}^{\theta}\right\}^{-1}\right]\right\}+\frac{\alpha \theta}{\beta} R_{D+1}^{*} e^{-(Q)^{\alpha}} Q^{\alpha} \xi_{Q}^{2 \theta-1} \log \xi_{Q}\left\{1-\xi_{Q}^{\theta}\right\}^{-2} \\
& +\left\{1-\xi_{Q}^{\theta}\right\}^{-1}\left\{\frac{\alpha}{\beta} R_{D+1}^{*} e^{-(Q)^{\alpha}} Q^{\alpha} \xi_{Q}^{\theta-1}\left\{1+\theta \log \xi_{Q}\right\}\right\}
\end{aligned}
$$

where, $\xi_{Q}=1-e^{-(Q)^{\alpha}}$ and $\xi_{U}=1-e^{-(U)^{\alpha}}$.

\section{Appendix B:}

The elements of the observed Fisher information matrix (11) based on the log-likelihood function (9) will be

$$
\begin{aligned}
& \frac{\partial^{2} l_{i}(\underline{\omega} \mid \underline{\mathrm{X}})}{\partial \alpha^{2}}=-\frac{S_{i}}{\alpha^{2}}-\sum_{j=1}^{S_{i}} U^{\alpha} \log U^{2}-(\theta-1) \sum_{j=1}^{S_{i}} e^{-(U)^{\alpha}}\left\{U^{\alpha} \log U\right\}^{2} \xi_{U}^{-1} \\
& \times\left\{1-U^{-\alpha}+e^{-(U)^{\alpha}} \xi_{U}^{-1}\right\}-\theta \sum_{j=1}^{S_{i}} R_{j} e^{-(U)^{\alpha}} U^{\alpha} \log U^{2} \xi_{U}^{\theta-1}\left\{1-\xi_{U}^{\theta}\right\}^{-1} \\
& \times\left\{1-U^{\alpha}+(\theta-1) e^{-(U)^{\alpha}} U^{\alpha} \xi_{U}^{-1}+\theta e^{-(U)^{\alpha}} U^{\alpha} \xi_{U}^{\theta-1}\left\{1-\xi_{U}^{\theta}\right\}^{-1}\right\} \\
& -\theta R_{D_{\tau}+1}^{*} e^{-(Q)^{\alpha}} Q^{\alpha} \log Q^{2} \xi_{Q}^{\theta-1}\left\{1-\xi_{Q}^{\theta}\right\}^{-1}\left\{1-Q^{\alpha}+(\theta-1) e^{-(Q)^{\alpha}} Q^{\alpha} \xi_{Q}^{-1}\right. \\
& \left.+\theta e^{-(Q)^{\alpha}} Q^{\alpha} \xi_{Q}^{\theta-1}\left\{1-\xi_{Q}^{\theta}\right\}^{-1}\right\} \\
& \frac{\partial^{2} l_{i}(\underline{\omega} \mid \underline{\mathrm{X}})}{\partial \beta^{2}}=\frac{\alpha S_{i}}{\beta^{2}}-\frac{\alpha(1+\alpha)}{\beta^{2}} \sum_{j=1}^{S_{i}} U^{\alpha}+\frac{\alpha(\theta-1)}{\beta^{2}} \sum_{j=1}^{S_{i}} e^{-(U)^{\alpha}} U^{\alpha} \xi_{U}^{-1}\left\{1+\alpha-U-e^{-(U)^{\alpha}} U^{\alpha} \xi_{U}^{-1}\right\} \\
& -\frac{\alpha \theta}{\beta} \sum_{j=1}^{S_{i}} R_{j} e^{-(U)^{\alpha}} U^{\alpha} \xi_{U}^{\theta-1}\left\{1-\xi_{U}^{\theta}\right\}^{-1}\left\{\frac{1}{\beta}+\frac{\alpha}{\beta}-\alpha U^{\alpha}+\frac{\alpha(\theta-1)}{\beta} e^{-(U)^{\alpha}} U^{\alpha} \xi_{U}^{-1}\right. \\
& \left.+\frac{\alpha \theta}{\beta} e^{-(U)^{\alpha}} U^{\alpha} \xi_{U}^{\theta-1}\left\{1-\xi_{U}^{\theta}\right\}^{-1}\right\}+\frac{\alpha \theta}{\beta} R_{D_{\tau}+1}^{*} e^{-(Q)^{\alpha}} Q^{\alpha} \xi_{Q}^{\theta-1}\left\{1-\xi_{Q}^{\theta}\right\}^{-1} \\
& \times\left\{\frac{\alpha}{\beta} Q^{\alpha}-\frac{\alpha}{\beta}-\frac{1}{\beta}+\frac{\alpha \theta}{\beta} e^{-(Q)^{\alpha}} Q^{\alpha} \xi_{Q}^{\theta-1}\left\{1-\xi_{Q}^{\theta}\right\}^{-1}-\frac{\alpha(\theta-1)}{\beta} e^{-(Q)^{\alpha}} Q^{\alpha} \xi_{Q}^{-1}\right\} .
\end{aligned}
$$




$$
\begin{aligned}
& \frac{\partial^{2} l_{i}(\underline{\omega} \mid \underline{\mathrm{X}})}{\partial \theta^{2}}=-\frac{S_{i}}{\theta^{2}}-\left\{\sum_{j=1}^{S_{i}} R_{j} \xi_{U}^{\theta} \log \xi_{U}^{2}\left\{1-\xi_{U}^{\theta}\right\}^{-1}\left[1+\xi_{U}^{\theta}\left\{1-\xi_{U}^{\theta}\right\}^{-1}\right]\right\} \\
& -R_{D_{\tau}+1}^{*} \xi_{Q}^{\theta} \log \xi_{Q}^{2}\left\{1-\xi_{Q}^{\theta}\right\}^{-1}\left\{1+\xi_{Q}^{\theta}\left\{1-\xi_{Q}^{\theta}\right\}^{-1}\right\} \text {. } \\
& \frac{\partial^{2} l_{i}(\underline{\omega} \mid \underline{\mathrm{X}})}{\partial \alpha \partial \theta}=\sum_{j=1}^{S_{i}} e^{-(U)^{\alpha}} U^{\alpha} \log U \xi_{U}^{-1}-\sum_{j=1}^{S_{i}} R_{j} e^{-(U)^{\alpha}} U^{\alpha} \log U \xi_{U}^{\theta-1}\left\{1-\xi_{U}^{\theta}\right\}^{-1} \\
& \times\left\{1+\theta \log \xi_{U}+\theta \xi_{U}^{\theta} \log \xi_{U}\left\{1-\xi_{U}^{\theta}\right\}^{-1}\right\}-\theta R_{D_{\tau}+1}^{*} e^{-(Q)^{\alpha}} Q^{\alpha} \log Q \xi_{Q}^{\theta-1}\left\{1-\xi_{Q}^{\theta}\right\}^{-1} \\
& \times\left\{1+\log \xi_{Q}+\xi_{Q}^{\theta} \log \xi_{Q}\left\{1-\xi_{Q}^{\theta}\right\}^{-1}\right\} . \\
& \frac{\partial^{2} l_{i}(\underline{\omega} \mid \underline{\mathrm{X}})}{\partial \beta \partial \alpha}=-\frac{S_{i}}{\beta}+\frac{1}{\beta} \sum_{j=1}^{S_{i}} U^{\alpha}\{1+\alpha \log U\}-\frac{(\theta-1)}{\beta} \sum_{j=1}^{S_{i}} e^{-(U)^{\alpha}} U^{\alpha} \xi_{U}^{-1} \\
& \times\left\{1+\alpha \log U\left[1-U^{\alpha}-e^{-(U)^{\alpha}} U^{\alpha} \xi_{U}^{-1}\right]\right\}+\frac{\theta}{\beta} \sum_{j=1}^{S_{i}} R_{j} e^{-(U)^{\alpha}} U^{\alpha} \xi_{U}^{\theta-1}\left\{1-\xi_{U}^{\theta}\right\}^{-1} \\
& \times\left\{1+\alpha \log U\left[1-U^{\alpha}+(\theta-1) e^{-(U)^{\alpha}} U^{\alpha} \xi_{U}^{-1}+\theta e^{-(U)^{\alpha}} U^{\alpha} \xi_{U}^{\theta-1}\left\{1-\xi_{U}^{\theta}\right\}^{-1}\right]\right\} \\
& +\frac{\theta}{\beta} R_{D_{\tau}+1}^{*} e^{-(Q)^{\alpha}} Q^{\alpha} \xi_{Q}^{\theta-1}\left\{1-\xi_{Q}^{\theta}\right\}^{-1}\left\{1+\alpha \log Q\left[1-Q^{\alpha}+(\theta-1) e^{-(Q)^{\alpha}} Q^{\alpha} \xi_{Q}^{-1}\right.\right. \\
& \left.\left.+\alpha \theta e^{-(Q)^{\alpha}} Q^{\alpha} \log Q \xi_{Q}^{\theta-1}\left\{1-\xi_{Q}^{\theta}\right\}^{-1}\right]\right\} .
\end{aligned}
$$

and

$$
\begin{aligned}
\frac{\partial^{2} l_{i}(\underline{\omega} \mid \underline{X})}{\partial \beta \partial \theta} & =-\frac{\alpha}{\beta} \sum_{j=1}^{S_{i}} e^{-(U)^{\alpha}} U^{\alpha} \xi_{U}^{-1}+\frac{\alpha}{\beta} \sum_{j=1}^{S_{i}} R_{j} e^{-(U)^{\alpha}} U^{\alpha} \xi_{U}^{\theta-1}\left\{1-\xi_{U}^{\theta}\right\}^{-1} \\
& \times\left\{1+\theta \log \xi_{U}\left[1+\xi_{U}^{\theta}\left\{1-\xi_{U}^{\theta}\right\}^{-1}\right]\right\}+\frac{\alpha}{\beta} R_{D_{\tau}+1}^{*} e^{-(Q)^{\alpha}} Q^{\alpha} \xi_{Q}^{\theta-1}\left\{1-\xi_{Q}^{\theta}\right\}^{-1} \\
& \times\left\{1+\theta \log \xi_{Q}\left[1+\xi_{Q}^{\theta}\left\{1-\xi_{Q}^{\theta}\right\}^{-1}\right]\right\} .
\end{aligned}
$$

where, $\xi_{Q}=1-e^{-(Q)^{\alpha}}$ and $\xi_{U}=1-e^{-(U)^{\alpha}}$. 